ESAIM: COCV 21 (2015) 138-164

DOI: $10.1051 / \mathrm{cocv} / 2014029$
ESAIM: Control, Optimisation and Calculus of Variations

www.esaim-cocv.org

\title{
ASYMPTOTIC BEHAVIOR OF THE APPROXIMATE CONTROLS FOR PARABOLIC EQUATIONS WITH INTERFACIAL CONTACT RESISTANCE*
}

\author{
Patrizia Donato ${ }^{1}$ and Editha C. $\mathrm{JOSE}^{2}$
}

\begin{abstract}
In this paper, we study the approximate control for a class of parabolic equations with rapidly oscillating coefficients in an $\varepsilon$-periodic composite with an interfacial contact resistance as well as its asymptotic behavior, as $\varepsilon \rightarrow 0$. The condition on the interface depends on a parameter $\gamma \in(-1,1]$. The case $\gamma=1$ is the most interesting one, and the more delicate, since the homogenized problem is given by coupled system of a P.D.E. and an O.D.E., giving rise to a memory effect. The variational approach to approximate controllability introduced by Lions in [J.-L. Lions. In Proc. of Jornadas Hispano-Francesas sobre Control de Sistemas Distribuidos, octubre 1990. Grupo de Análisis Matemático Aplicado de la University of Malaga, Spain (1991) 77-87] lead us to the construction of the control as the solution of a related transposed problem. The final data of this problem is the unique minimum point of a suitable functional $J_{\varepsilon}$. The more interesting result of this study proves that the control and the corresponding solution of the $\varepsilon$-problem converge respectively to a control of the homogenized problem and to the corresponding solution. The main difficulties here are to find the appropriate limit functionals for the control of the homogenized system and to identify the limit of the controls.
\end{abstract}

Mathematics Subject Classification. 35B27, 35Q93, 93B05.

Received August 29, 2013. Revised May 8, 2014.

Published online December 3, 2014.

\section{INTRODUCTION}

The aim of this study is to answer two questions about the approximate controllability of a linear parabolic problem with oscillating coefficients on an $\varepsilon$-periodic two-component composite given by $\Omega=\Omega_{1 \varepsilon} \cup \overline{\Omega_{2 \varepsilon}}$. The component $\Omega_{1 \varepsilon}$ is connected while $\Omega_{2 \varepsilon}$ is a disconnected union of $\varepsilon$-periodic translated sets of $\varepsilon Y_{2}$. On the other hand, $\Gamma_{\varepsilon}$ is the interface separating the two components with $\partial \Omega \cap \Gamma_{\varepsilon}=\emptyset$ (see Fig. 1). On the interface, a jump

\footnotetext{
Keywords and phrases. Approximate controllability of parabolic equation, homogenization in a two-component domain with a periodic interface, jump condition on the interface depending on a parameter.

* The second author is grateful for the financial support extended by Federation Normandie Mathématiques during her research visit at the Laboratoire de Mathématiques Raphaël Salem, Université de Rouen.

1 Normandie Université, Université de Rouen, Laboratoire de Mathématiques Raphaël Salem, CNRS, UMR 6085, Avenue de l'Université, BP 12, 76801 Saint-Étienne du Rouvray cedex, France. Patrizia.Donato@univ-rouen.fr

2 Institute of Mathematical Sciences and Physics, UP Los Baños, College, Los Baños, Laguna, Philippines.

ecjose@uplb.edu.ph
} 


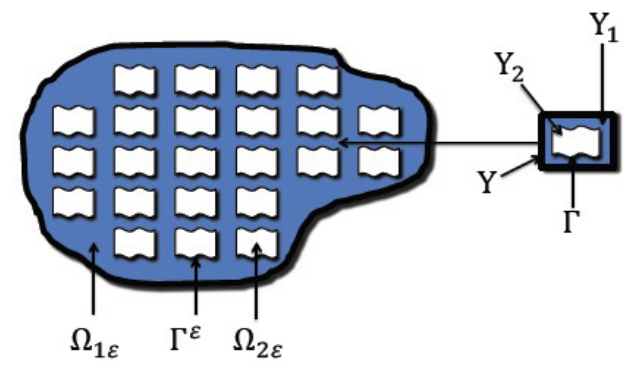

Figure 1. The two-component domain.

of the solution is prescribed so that it is proportional to the conormal derivative via a parameter $\gamma \in(-1,1]$, meanwhile, a Dirichlet condition is imposed on the exterior boundary $\partial \Omega$.

This problem models the heat diffusion in a two-component composite with an imperfect contact on the interface (see [5] for a physical justification of the model) and its limit problem (as $\varepsilon \rightarrow 0$ ) describing the effective thermal conductivity of medium, under the influence of the contact barrier.

Let us recall that by definition one has approximate controllability if the set of reachable final states is dense in $\mathrm{L}^{2}(\Omega)$. The first question deals with the existence of an approximate control of the $\varepsilon$-problem. If such a control exists, the second question, which is the more interesting one, is: do the control and the corresponding solution of the $\varepsilon$-problem converge $($ as $\varepsilon \rightarrow 0$ ) respectively to a control of the homogenized problem and to the corresponding solution? In Section 3, we were able to answer both of these questions.

It is already known from previous studies by the authors in $[6,10]$ (see also [8]) that the asymptotic behavior of the $\varepsilon$-problem differs in terms of the homogenized problems in the two cases $-1<\gamma<1$ and $\gamma=1$. The second one being the most complicated and interesting one, since the limit problem is a coupled system of a P.D.E. and an O.D.E., gives rise to what is called a memory effect. In Section 2 we give the precise setting of the problem and recall the homogenization and corrector results from $[6,10]$.

Then, in Section 3 we state Theorem 3.2, which provides the approximate controllability of the $\varepsilon$-problem. In order to seek an answer to the second question, we also show the approximate controllability of the two different homogenized problems corresponding to the case $-1<\gamma<1$ (Thm. 3.3) and $\gamma=1$ (Thm. 3.4). This is followed by the main convergence result of this study (Thm. 3.5), which positively answers the second question.

Following an idea introduced by Lions in [11], the construction of the control relies on the definition of a suitable functional such that the control can be obtained as the solutions of a related transposed problem having as final data the (unique) minimum point of the functional. We adapt some ideas used in $[7,9,13,14]$ to our case of a jump condition on the interface between the two components.

The unique continuation property due to Saut and Scheurer [12] plays an important role to guarantee the existence of a minimizer of the functionals for the $\varepsilon$-problem as well as for the homogenized problem of the case $-1<\gamma<1$. For the homogenized problem when $\gamma=1$, we need a special version of this property, which is a new result and has been proved by Ammar Kohdja [1]. We added here its proof in the Appendix.

The existence of the controls of the homogenized problems and of the $\varepsilon$-problem is proved in Sections 4 and 5 , respectively. We show first in details the controllability of the homogenized problems which is more delicate, in particular that corresponding to the case $\gamma=1$. Afterwards, we prove the approximate controllability for $\varepsilon$-problem detailing only the specific points.

Finally, in Section 6 we prove the main result of this study, which concerns the asymptotic behavior of the $\varepsilon$-controllability problem. To this end, one of the main difficulties is to identify the limit of the controls. This is done in Theorem 6.4, which provided a uniform estimate in $\varepsilon$ for the unique minimum point of $J_{\varepsilon}$, as well as some suitable properties of the functionals. 
Let us mention here that one of the main difficulties is to find the appropriate limit functionals, which can allow to give a positive answer to the second question. Although the $\varepsilon$-functional is defined on functions of $\mathrm{L}^{2}(\Omega)$, the limit functionals for the two cases need to be defined on couples of functions of $\mathrm{L}^{2}(\Omega)$, due to the structure of the domain. This is a technical point, which is related to the fact that if a sequence converges to a function in $\mathrm{L}^{2}(\Omega)$, the limit of the restriction to each component do not converge to $\theta_{i}$ (the proportion of material) multiplied by that function.

As usual, in the spirit of Gamma-convergence, one has to prove limit equalities (here given in Props. (6.6) and (6.7)) and a liminf inequality (proved in Prop. (6.9)). To prove the equalities, one needs to apply to the auxiliary problem the corrector results given in Section 2, which need some stronger assumptions than the homogenization results. We also emphasize that the corrector results are needed because we assume that the initial data may not be zero.

In particular, when proving that the limit of the minimum points of $J_{\varepsilon}$ converges to the minimum point of $J_{0}$ for the case $-1<\gamma<1$, with the aid of a (new) compactness result given in Proposition 2.4, we are able to derive a stronger convergence of the controls. Although this result does not hold for the case $\gamma=1$, we are still able to describe the behavior of the controls in the latter case, where corrector results are essential even in the case of zero initial data.

\section{Preliminaries}

\subsection{Position of the problem}

In this work, $\Omega$ is a connected bounded open set of $\left.\mathbb{R}^{n}(n \geq 2), Y=\right] 0, \ell_{1}[\times \ldots \times] 0, \ell_{n}[$ is the reference cell, and $\{\varepsilon\}$ is a sequence of positive real numbers that converges to zero.

Let $Y_{1}$ and $Y_{2}$ be two nonempty open sets such that $Y=Y_{1} \cup \overline{Y_{2}}$, with $Y_{1}$ connected and $\Gamma:=\partial Y_{2}$ Lipschitz continuous. We define for any $k \in \mathbb{Z}^{n}$, the translated sets $Y_{i}^{k}$ and $\Gamma_{k}$ as follows:

$$
Y_{i}^{k}:=k_{\ell}+Y_{i}, \quad \Gamma_{k}:=k_{\ell}+\Gamma \quad \text { where } \quad k_{\ell}=\left(k_{1} \ell_{1}, \ldots, k_{n} \ell_{n}\right) \quad \text { and } \quad i=1,2
$$

and for any given $\varepsilon$, we set $K_{\varepsilon}:=\left\{k \in \mathbb{Z}^{n} \mid \varepsilon Y_{i}^{k} \cap \Omega \neq \emptyset, i=1,2\right\}$. We then define the two components of $\Omega$ and the interface respectively by

$$
\Omega_{i \varepsilon}:=\Omega \cap\left\{\bigcup_{k \in K_{\varepsilon}} \varepsilon Y_{i}^{k}\right\}, \quad i=1,2 \quad \text { and } \quad \Gamma_{\varepsilon}:=\partial \Omega_{2 \varepsilon} .
$$

We assume that

$$
\partial \Omega \cap\left(\bigcup_{k \in \mathbb{Z}^{n}}\left(\varepsilon \Gamma_{k}\right)\right)=\emptyset .
$$

By construction, $\Omega$ is decomposed into two components, $\Omega=\Omega_{1 \varepsilon} \cup \overline{\Omega_{2 \varepsilon}}$, where $\Omega_{1 \varepsilon}$ is connected, $\Omega_{2 \varepsilon}$ is a disconnected union of $\varepsilon$-periodic translated sets of $\varepsilon Y_{2}$ and $\Gamma_{\varepsilon}$ is the interface separating the two components with $\partial \Omega \cap \Gamma_{\varepsilon}=\emptyset$. Figure 1 shows the two-component domain.

In the following we denote by

$-\sim$ the zero extension to the whole of $\Omega$ of functions defined on $\Omega_{1 \varepsilon}$ or $\Omega_{2 \varepsilon}$,

$-\chi_{E}$ the characteristic function of any measurable set $E \subset \mathbb{R}^{n}$.

$-m_{E}(v)=\frac{1}{|E|} \int_{E} v \mathrm{~d} x$ the average on $E$ of any function $v \in \mathrm{L}^{1}(E)$.

Let us recall (see for instance [3]) that as $\varepsilon \rightarrow 0$, for $i=1,2$,

$$
\chi_{\Omega_{i \varepsilon}} \rightarrow \theta_{i}:=\frac{\left|Y_{i}\right|}{|Y|} \text { weakly in } \mathrm{L}^{2}(\Omega),
$$

$\theta_{i}$ being the proportion of the material occupying $\Omega_{i \varepsilon}$. 
Let now $\omega$ be a given open non-empty subset of $\Omega$, and set

$$
\omega_{i \varepsilon}=\omega \cap \Omega_{i \varepsilon}, \quad i=1,2 .
$$

The aim of this paper is to study the approximate controllability and the asymptotic behavior as $\varepsilon \rightarrow 0$, of the following problem:

$$
\begin{cases}u_{1 \varepsilon}{ }^{\prime}-\operatorname{div}\left(A\left(\frac{x}{\varepsilon}\right) \nabla u_{1 \varepsilon}\right)=\chi_{\omega_{1 \varepsilon}} \varphi_{1 \varepsilon} & \text { in } \Omega_{1 \varepsilon} \times(0, T), \\ u_{2 \varepsilon}{ }^{\prime}-\operatorname{div}\left(A\left(\frac{x}{\varepsilon}\right) \nabla u_{2 \varepsilon}\right)=\chi_{\omega_{2 \varepsilon}} \varphi_{2 \varepsilon} & \text { in } \Omega_{2 \varepsilon} \times(0, T), \\ A\left(\frac{x}{\varepsilon}\right) \nabla u_{1 \varepsilon} \cdot n_{1 \varepsilon}=-A\left(\frac{x}{\varepsilon}\right) \nabla u_{2 \varepsilon} \cdot n_{2 \varepsilon} & \text { on } \Gamma_{\varepsilon} \times(0, T), \\ A\left(\frac{x}{\varepsilon}\right) \nabla u_{1 \varepsilon} \cdot n_{1 \varepsilon}=-\varepsilon^{\gamma} h\left(\frac{x}{\varepsilon}\right)\left(u_{1 \varepsilon}-u_{2 \varepsilon}\right) & \text { on } \Gamma_{\varepsilon} \times(0, T), \\ u_{\varepsilon}=0 & \text { on } \partial \Omega \times(0, T), \\ u_{1 \varepsilon}(x, 0)=\left.U_{\varepsilon}^{0}\right|_{\Omega_{1 \varepsilon}} & \text { in } \Omega_{1 \varepsilon}, \\ u_{2 \varepsilon}(x, 0)=\left.U_{\varepsilon}^{0}\right|_{\Omega_{2 \varepsilon}} & \text { in } \Omega_{2 \varepsilon},\end{cases}
$$

where $n_{i \varepsilon}$ is the unitary outward normal to $\Omega_{i \varepsilon}(i=1,2), U_{\varepsilon}^{0}$ is given in $\mathrm{L}^{2}(\Omega)$, and $\gamma \in(-1,1]$.

In the sequel, we assume that

$$
\omega_{1 \varepsilon} \neq \emptyset, \quad \text { for any } \varepsilon,
$$

which is not restrictive for our aim, since for a given $\omega$ this is always true for $\varepsilon$ sufficiently small.

We suppose that $A$ is a $Y$-periodic symmetric $n \times n$-matrix field in $\mathcal{M}(\alpha, \beta, \Omega)$, that is,

$$
\left\{\begin{array}{l}
\text { (i) } A \in\left(\mathrm{L}^{\infty}(Y)\right)^{n^{2}} \quad \text { and } \quad a_{i j}=a_{j i}, 1 \leq i, j \leq n, \\
\text { (ii) }(A(x) \lambda, \lambda) \geq \alpha|\lambda|^{2}, \quad|A(x) \lambda| \leq \beta|\lambda|,
\end{array}\right.
$$

for every $\lambda \in \mathbb{R}^{N}$ and a.e. in $\Omega$ where $\alpha, \beta \in \mathbb{R}$ with $0<\alpha<\beta$. Moreover, we suppose that

$$
\begin{gathered}
h \in \mathrm{L}^{\infty}(\Gamma), \quad \exists h_{0} \in \mathbb{R} \quad \text { such that } 0<h_{0}<h(y), \quad y \text { a.e. in } \Gamma, \\
U_{\varepsilon}^{0} \in \mathrm{L}^{2}(\Omega), \quad \varphi_{i \varepsilon} \in \mathrm{L}^{2}\left(0, T ; \mathrm{L}^{2}(\Omega)\right),(i=1,2) .
\end{gathered}
$$

In the following, we set

$$
A\left(\frac{x}{\varepsilon}\right)=A^{\varepsilon}(x), \quad h\left(\frac{x}{\varepsilon}\right)=h^{\varepsilon}(x) .
$$

The approximate controllability problem for system (2.2) reads:

Given $w_{\varepsilon} \in \mathrm{L}^{2}(\Omega), \delta_{1}>0$ and $\delta_{2}>0$, does a control $\widehat{\varphi}_{\varepsilon}=\left(\widehat{\varphi}_{1 \varepsilon}, \widehat{\varphi}_{2 \varepsilon}\right) \in \mathrm{L}^{2}\left(\Omega_{1 \varepsilon}\right) \times \mathrm{L}^{2}\left(\Omega_{2 \varepsilon}\right)$ exist such that the solution $u_{\varepsilon}=\left(u_{1 \varepsilon}, u_{2 \varepsilon}\right)$ of $(2.2)$ verifies the estimates

$$
\left\{\begin{array}{l}
\text { (i) }\left\|u_{1 \varepsilon}(T)-w_{\varepsilon}\right\|_{L^{2}\left(\Omega_{1 \varepsilon}\right)} \leq \delta_{1} \\
\text { (ii) }\left\|u_{2 \varepsilon}(T)-w_{\varepsilon}\right\|_{L^{2}\left(\Omega_{2 \varepsilon}\right)} \leq \delta_{2} ?
\end{array}\right.
$$

Another interesting question is:

If there is such a control, do the control and the corresponding solution of (2.2) converge (as $\varepsilon \rightarrow 0$ ) to a control of the homogenized problem and to the corresponding solution, respectively?

In this paper, we give positive answers to both questions. Concerning the first question we need the additional assumption that

$$
A \in\left(\mathrm{C}^{1}(\bar{Y})\right)^{n^{2}},
$$


since, as usually in the literature for this kind of problem, we need to make use some unique-continuation properties, as that due to Saut and Scheurer [12]. In order to answer the second question, we will make use of some homogenization and corrector results for system $(2.2)$ which were studied in $[6,10]$.

Let us mention that pioneer results on approximate controllability can be traced back from the works of Lions [11]. For the asymptotic behavior of the approximate controllability problem of linear parabolic equations we refer to [14] (see also [13]) for the case of a fixed domain and to [7] for the case in a perforated domain.

\subsection{Recall of the asymptotic behavior of the $\varepsilon$-problem}

We introduce first the functional spaces

$$
V^{\varepsilon}:=\left\{v_{1} \in \mathrm{H}^{1}\left(\Omega_{1 \varepsilon}\right) \mid v_{1}=0 \text { on } \partial \Omega\right\} \text { equipped with the norm }\left\|v_{1}\right\|_{V^{\varepsilon}}:=\left\|\nabla v_{1}\right\|_{\mathrm{L}^{2}\left(\Omega_{1 \varepsilon}\right)}
$$

and

$$
W^{\varepsilon}:=\left\{v=\left(v_{1}, v_{2}\right) \in \mathrm{L}^{2}\left(0, T ; V^{\varepsilon}\right) \times \mathrm{L}^{2}\left(0, T ; \mathrm{H}^{1}\left(\Omega_{2 \varepsilon}\right)\right) \mid v^{\prime} \in \mathrm{L}^{2}\left(0, T ;\left(V^{\varepsilon}\right)^{\prime}\right) \times \mathrm{L}^{2}\left(0, T ;\left(\mathrm{H}^{1}\left(\Omega_{2 \varepsilon}\right)\right)^{\prime}\right)\right\},
$$

equipped with the norm

$$
\|v\|_{W^{\varepsilon}}=\left\|v_{1}\right\|_{\mathrm{L}^{2}\left(0, T ; V^{\varepsilon}\right)}+\left\|v_{2}\right\|_{\mathrm{L}^{2}\left(0, T ; \mathrm{H}^{1}\left(\Omega_{2 \varepsilon}\right)\right)}+\left\|v_{1}^{\prime}\right\|_{\mathrm{L}^{2}\left(0, T ;\left(V^{\varepsilon}\right)^{\prime}\right)}+\left\|v_{2}^{\prime}\right\|_{\mathrm{L}^{2}\left(0, T ;\left(\mathrm{H}^{1}\left(\Omega_{2 \varepsilon}\right)\right)^{\prime}\right)} .
$$

It has been shown in [10] that problem $(2.2)$ has a unique solution $u^{\varepsilon}=\left(u_{1 \varepsilon}, u_{2 \varepsilon}\right) \in W^{\varepsilon}$ and its asymptotic behavior was studied in $[6,10]$.

Remark 2.1. In this paper, $\mathrm{L}^{2}\left(\Omega_{1 \varepsilon}\right) \times \mathrm{L}^{2}\left(\Omega_{2 \varepsilon}\right)$ will be equipped with the usual product norm, that is,

$$
\forall\left(w_{1}, w_{2}\right) \in \mathrm{L}^{2}\left(\Omega_{1 \varepsilon}\right) \times \mathrm{L}^{2}\left(\Omega_{2 \varepsilon}\right), \quad\left\|\left(w_{1}, w_{2}\right)\right\|_{\mathrm{L}^{2}\left(\Omega_{1 \varepsilon}\right) \times \mathrm{L}^{2}\left(\Omega_{2 \varepsilon}\right)}=\left(\left\|w_{1}\right\|_{\mathrm{L}^{2}\left(\Omega_{1 \varepsilon}\right)}^{2}+\left\|w_{2}\right\|_{\mathrm{L}^{2}\left(\Omega_{2 \varepsilon}\right)}^{2}\right)^{\frac{1}{2}} .
$$

Observe that the map $\Phi: v \in \mathrm{L}^{2}(\Omega) \rightarrow\left(\left.v\right|_{\Omega_{1 \varepsilon}},\left.v\right|_{\Omega_{2 \varepsilon}}\right) \in \mathrm{L}^{2}\left(\Omega_{1 \varepsilon}\right) \times \mathrm{L}^{2}\left(\Omega_{2 \varepsilon}\right)$ is a bijective isometry since

$$
\|v\|_{\mathrm{L}^{2}(\Omega)}^{2}=\|v\|_{\mathrm{L}^{2}\left(\Omega_{1 \varepsilon}\right)}^{2}+\|v\|_{\mathrm{L}^{2}\left(\Omega_{2 \varepsilon}\right)}^{2}, \quad \text { for every } v \in \mathrm{L}^{2}(\Omega) .
$$

Notations. In view of Remark 2.1, in the sequel, we identify $v \in \mathrm{L}^{2}(\Omega)$ with $\left(\left.v\right|_{\Omega_{1 \varepsilon}},\left.v\right|_{\Omega_{2 \varepsilon}}\right) \in \mathrm{L}^{2}\left(\Omega_{1 \varepsilon}\right) \times \mathrm{L}^{2}\left(\Omega_{2 \varepsilon}\right)$. When no confusion arises, we will simply write $v$ instead of $\left.v\right|_{\Omega_{i \varepsilon}}, i=1,2$. Also, we will use the superscript 0 for functions taken as initial or final data in different parabolic problems, as for instance, $U^{0} \in \mathrm{L}^{2}(\Omega)$ in (3.7).

We will make use of some homogenization and corrector results proved in $[6,10]$ that we recall below, for the reader's convenience.

Theorem 2.2 [10]. For $-1<\gamma \leq 1$, suppose that $A^{\varepsilon}$ and $h^{\varepsilon}$ satisfy $(2.4)-(2.7)$. Let $z_{\varepsilon}=\left(z_{1 \varepsilon}, z_{2 \varepsilon}\right)$ be the solution of the following problem:

$$
\begin{cases}z_{i \varepsilon}{ }^{\prime}-\operatorname{div}\left(A^{\varepsilon} \nabla z_{i \varepsilon}\right)=g_{i \varepsilon} & \text { in } \Omega_{i \varepsilon} \times(0, T), i=1,2, \\ A^{\varepsilon} \nabla z_{1 \varepsilon} \cdot n_{1 \varepsilon}=-A^{\varepsilon} \nabla z_{2 \varepsilon} \cdot n_{2 \varepsilon} & \text { on } \Gamma_{\varepsilon} \times(0, T), \\ A^{\varepsilon} \nabla z_{1 \varepsilon} \cdot n_{1 \varepsilon}=-\varepsilon^{\gamma} h^{\varepsilon}\left(z_{1 \varepsilon}-z_{2 \varepsilon}\right) & \text { on } \Gamma_{\varepsilon} \times(0, T), \\ z_{\varepsilon}=0 & \text { on } \partial \Omega \times(0, T), \\ z_{i \varepsilon}(x, 0)=\left.Z_{\varepsilon}^{0}\right|_{\Omega_{i \varepsilon}} & \text { in } \Omega_{i \varepsilon}, i=1,2,\end{cases}
$$

where $Z_{\varepsilon}^{0} \in \mathrm{L}^{2}(\Omega)$ and $\left(g_{1 \varepsilon}, g_{2 \varepsilon}\right) \in\left[\mathrm{L}^{2}\left(0, T ; \mathrm{L}^{2}(\Omega)\right)\right]^{2}$. If

$$
\left\{\begin{array}{l}
(\mathrm{i}) \quad\left(\chi_{\Omega_{1 \varepsilon}} Z_{\varepsilon}^{0}, \chi_{\Omega_{2 \varepsilon}} Z_{\varepsilon}^{0}\right) \rightarrow\left(\theta_{1} Z_{1}^{0}, \theta_{2} Z_{2}^{0}\right) \quad \text { weakly in }\left[\mathrm{L}^{2}(\Omega)\right]^{2}, \\
\text { (ii) }\left(\chi_{\Omega_{1 \varepsilon}} g_{1 \varepsilon}, \chi_{\Omega_{1 \varepsilon}} g_{2 \varepsilon}\right) \rightarrow\left(\theta_{1} g_{1}, \theta_{2} g_{2}\right) \quad \text { weakly in }\left[\mathrm{L}^{2}\left(0, T ; \mathrm{L}^{2}(\Omega)\right)\right]^{2},
\end{array}\right.
$$


then there exists a linear continuous extension operator $P_{1}^{\varepsilon} \in \mathcal{L}\left(\mathrm{L}^{2}\left(0, T ; V^{\varepsilon}\right) ; \mathrm{L}^{2}\left(0, T ; \mathrm{H}_{0}^{1}(\Omega)\right)\right) \cap$ $\mathcal{L}\left(\mathrm{L}^{2}\left(0, T ; \mathrm{L}^{2}\left(\Omega_{1 \varepsilon}\right)\right) ; \mathrm{L}^{2}\left(0, T ; \mathrm{L}^{2}(\Omega)\right)\right)$ such that

$$
\begin{cases}\text { (i) } \quad P_{1}^{\varepsilon} z_{1 \varepsilon} \rightarrow z_{1} & \text { weakly in } \mathrm{L}^{2}\left(0, T ; \mathrm{H}_{0}^{1}(\Omega)\right), \\ \text { (ii) } \widetilde{z_{1 \varepsilon}} \rightarrow \theta_{1} z_{1} & \text { weakly* in } \mathrm{L}^{\infty}\left(0, T ; \mathrm{L}^{2}(\Omega)\right), \\ \text { (iii) } \widetilde{z_{2 \varepsilon}} \rightarrow z_{2} & \text { weakly* in } \mathrm{L}^{\infty}\left(0, T ; \mathrm{L}^{2}(\Omega)\right), \\ \text { (iv) } \varepsilon^{\frac{\gamma}{2}}\left\|z_{1 \varepsilon}-z_{2 \varepsilon}\right\|_{\mathrm{L}^{2}\left(0, T ; \mathrm{L}^{2}\left(\Gamma_{\varepsilon}\right)\right)}<c, & \end{cases}
$$

where $\sim$ denotes the zero extension to the whole of $\Omega$. Furthermore,

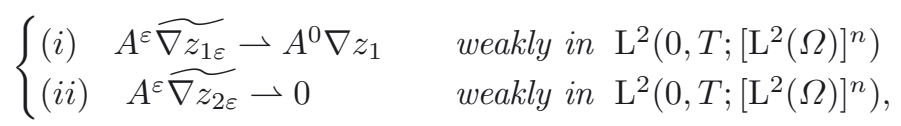

where $A^{0} \lambda:=m_{Y}\left(A \widetilde{\widehat{w}_{\lambda}}\right)$, the function $\widehat{w}_{\lambda} \in \mathrm{H}^{1}\left(Y_{1}\right)$ being for any $\lambda \in \mathbb{R}$, the unique solution of the problem

$$
\left\{\begin{array}{l}
-\operatorname{div}\left(A \nabla \widehat{w}_{\lambda}\right)=0 \quad \text { in } Y_{1}, \\
\left(A \nabla \widehat{w}_{\lambda}\right) \cdot n_{1}=0 \quad \text { in } \Gamma \\
\widehat{w}_{\lambda}-\lambda \cdot y Y \text {-periodic and } m_{Y_{1}}\left(\widehat{w}_{\lambda}-\lambda \cdot y\right)=0 .
\end{array}\right.
$$

The homogenized problems satisfied by the couple $\left(z_{1}, z_{2}\right)$ are different for the two cases $-1<\gamma<1$ and $\gamma=1$. Case $-\mathbf{1}<\boldsymbol{\gamma}<\mathbf{1}$ : The function $z_{2}$ is given by $z_{2}=\theta_{2} z_{1}$ and $z_{1} \in \mathrm{C}^{0}\left([0, T] ; \mathrm{L}^{2}(\Omega)\right) \cap \mathrm{L}^{2}\left(0, T ; \mathrm{H}_{0}^{1}(\Omega)\right)$ with $z_{1}^{\prime} \in \mathrm{L}^{2}\left(0, T ; \mathrm{H}^{-1}(\Omega)\right)$ is the unique solution of the homogenized problem

$$
\begin{cases}z_{1}^{\prime}-\operatorname{div}\left(A^{0} \nabla z_{1}\right)=\theta_{1} g_{1}+\theta_{2} g_{2} & \text { in } \Omega \times(0, T), \\ z_{1}=0 & \text { on } \partial \Omega \times(0, T), \\ z_{1}(0)=\theta_{1} Z_{1}^{0}+\theta_{2} Z_{2}^{0} & \text { in } \Omega .\end{cases}
$$

Case $\gamma=1$ : The pair $\left(z_{1}, z_{2}\right) \in \mathrm{C}^{0}\left([0, T] ; \mathrm{L}^{2}(\Omega)\right) \cap \mathrm{L}^{2}\left(0, T ; \mathrm{H}_{0}^{1}(\Omega)\right) \times \mathrm{C}^{0}\left([0, T] ; \mathrm{L}^{2}(\Omega)\right)$ with $z_{1}^{\prime} \in$ $\mathrm{L}^{2}\left(0, T ; \mathrm{H}^{-1}(\Omega)\right)$ is the unique solution of the coupled system

$$
\begin{cases}\theta_{1} z_{1}^{\prime}-\operatorname{div}\left(A^{0} \nabla z_{1}\right)+c_{h}\left(\theta_{2} z_{1}-z_{2}\right)=\theta_{1} g_{1} & \text { in } \Omega \times(0, T), \\ z_{2}^{\prime}-c_{h}\left(\theta_{2} z_{1}-z_{2}\right)=\theta_{2} g_{2} & \text { in } \Omega \times(0, T), \\ z_{1}=0 & \text { on } \partial \Omega \times(0, T), \\ z_{1}(0)=Z_{1}^{0}, z_{2}(0)=\theta_{2} Z_{2}^{0} & \text { in } \Omega,\end{cases}
$$

where $c_{h}=\frac{1}{\left|Y_{2}\right|} \int_{\Gamma} h(y) \mathrm{d} \sigma_{y}$.

Remark 2.3. The homogenized matrix $A^{0}$ is that obtained by Cioranescu and Saint Jean Paulin in [4] for the Laplace problem in a perforated domain with a Neumann condition on the boundary of the holes.

As proved in [10], in the case $\gamma=1$, solving the ODE in (2.17) and replacing $z_{2}$ in the PDE shows that $z_{1}$ satisfies an equation of the form

$$
\theta_{1} z_{1}^{\prime}-\operatorname{div}\left(A_{\gamma}^{0} \nabla z_{1}\right)+c_{h} \theta_{2} z_{1}-c_{h}^{2} \theta_{2} \int_{0}^{t} \mathcal{K}(t, s) z_{1}(s) \mathrm{d} s=F(x, t),
$$

with $\mathcal{K}$ an exponential kernel, giving rise to a memory effect.

In this paper, we complete the weak convergences stated in (2.14) by a strong convergence result given in the proposition below. This allows us to improve some convergences of the approximate controls when $-1<\gamma<1$. Let us emphasize that the results below do not hold for $\gamma=1$. 
Proposition 2.4. Let $-1<\gamma<1$. Under the assumptions of Theorem 2.2, let $z_{\varepsilon}=\left(z_{1 \varepsilon}, z_{2 \varepsilon}\right)$ and $z_{1}$ be the solutions of (2.12) and (2.16), respectively. Then,

$$
\widetilde{z_{1 \varepsilon}}+\widetilde{z_{2 \varepsilon}}=z_{\varepsilon} \rightarrow z_{1} \quad \text { in } \mathrm{L}^{2}\left(0, T ; \mathrm{L}^{2}(\Omega)\right) .
$$

Proof. When $\gamma<1$ from Proposition 3.7 of [10], one has

$$
\left\|P_{1}^{\varepsilon} z_{1 \varepsilon}-z_{2 \varepsilon}\right\|_{\mathrm{L}^{2}\left(0, T ; \mathrm{L}^{2}\left(\Omega_{2 \varepsilon}\right)\right)} \rightarrow 0 .
$$

On the other hand, since

$$
P_{1}^{\varepsilon} z_{1 \varepsilon}=\widetilde{z_{1 \varepsilon}}+\widetilde{z_{2 \varepsilon}}+\left(\chi_{\Omega_{2 \varepsilon}} P_{1}^{\varepsilon} z_{1 \varepsilon}-\widetilde{z_{2 \varepsilon}}\right)
$$

we have

$$
\int_{0}^{T} \int_{\Omega}\left|z_{\varepsilon}-z_{1}\right|^{2} \mathrm{~d} x \mathrm{~d} t \leq 2\left[\int_{0}^{T} \int_{\Omega}\left|P_{1}^{\varepsilon} z_{1 \varepsilon}-z_{1}\right|^{2} \mathrm{~d} x \mathrm{~d} t+\int_{0}^{T} \int_{\Omega_{2 \varepsilon}}\left|P_{1}^{\varepsilon} z_{1 \varepsilon}-z_{2 \varepsilon}\right|^{2} \mathrm{~d} x \mathrm{~d} t\right] .
$$

Hence, due to (2.19), in order to show (2.18) it is enough to prove that $P_{1}^{\varepsilon} z_{1 \varepsilon} \rightarrow z_{1}$ in $\mathrm{L}^{2}\left(0, T ; \mathrm{L}^{2}(\Omega)\right)$ and to do that, in view of $(2.14)$ and classical compactness results it suffices to prove that $\left(P_{1}^{\varepsilon} z_{1 \varepsilon}\right)^{\prime}$ is bounded in $\mathrm{L}^{2}\left(0, T ; \mathrm{H}^{-1}(\Omega)\right)$. Let us show first that

$$
\left(\chi_{\Omega_{2 \varepsilon}} P_{1}^{\varepsilon} z_{1 \varepsilon}-\widetilde{z_{2 \varepsilon}}\right)^{\prime} \rightarrow 0 \quad \text { weakly in } \mathrm{L}^{2}\left(0, T ; \mathrm{H}^{-1}(\Omega)\right)
$$

Indeed, for any $\varphi \in \mathcal{D}((0, T) \times \Omega)$ one has

$$
\left|<\left(\chi_{\Omega_{2 \varepsilon}} P_{1}^{\varepsilon} z_{1 \varepsilon}-\widetilde{z_{2 \varepsilon}}\right)^{\prime}, \varphi>_{\mathrm{L}^{2}\left([0, T] ; \mathrm{H}^{-1}(\Omega)\right), \mathrm{L}^{2}\left([0, T] ; \mathrm{H}_{0}^{1}(\Omega)\right)}\right|=\left|\int_{0}^{T} \int_{\Omega_{2 \varepsilon}}\left(P_{1}^{\varepsilon} z_{1 \varepsilon}-z_{2 \varepsilon}\right) \varphi^{\prime} \mathrm{d} x \mathrm{~d} t\right| .
$$

Since the right-hand side of this equality goes to zero in view of (2.19), this implies (2.21).

Let us recall now that from Theorem 4.7 of [6], the sequence $\left\{\widetilde{z_{1 \varepsilon}{ }^{\prime}}+\widetilde{z_{2 \varepsilon}}{ }^{\prime}\right\}$ is bounded in $\mathrm{L}^{2}\left(0, T ; \mathrm{H}^{-1}(\Omega)\right)$. This together with (2.21) implies the boundedness of $\left(P_{1}^{\varepsilon} z_{1 \varepsilon}\right)^{\prime}$ in $\mathrm{L}^{2}\left(0, T ; \mathrm{H}^{-1}(\Omega)\right)$ and concludes the proof, since from $(2.20),\left(P_{1}^{\varepsilon} z_{1 \varepsilon}\right)^{\prime}={\widetilde{z_{1 \varepsilon}}}^{\prime}+{\widetilde{z_{2 \varepsilon}}}^{\prime}+\left(\chi_{\Omega_{2 \varepsilon}} P_{1}^{\varepsilon} z_{1 \varepsilon}-z_{2 \varepsilon}\right)^{\prime}$.

Let us recall now the corrector results proved in [6], which are also different for the two cases. These corrector results were made possible by imposing stronger assumptions on the data.

If $\left(e_{j}\right)_{j=1, \ldots, n}$ is the canonical basis of $\mathbb{R}$ and $\widehat{w}_{j}$ is the solution of $(2.15)$ written for $\lambda=e_{j}, j=1, \ldots, n$, the corrector matrix $C^{\varepsilon}=\left(C_{i j}^{\varepsilon}\right)_{1 \leq i, j \leq n}$ is defined, for $i, j=1, \ldots, n$, by

$$
C_{i j}(y):=\frac{\partial \widehat{w}_{j}}{\partial y_{i}}(y), \quad \text { a.e. on } Y_{1}, \quad C_{i j}^{\varepsilon}(x)=\widetilde{C_{i j}}\left(\frac{x}{\varepsilon}\right) \text { a.e. on } \Omega .
$$

Theorem 2.5 [6]. Under assumptions $(2.4)-(2.7)$, let $z_{\varepsilon}=\left(z_{1 \varepsilon}, z_{2 \varepsilon}\right)$ be the solution of (2.12).

Case $-\mathbf{1}<\gamma<\mathbf{1}$ : Assume that the data $g_{i \varepsilon} \in \mathrm{L}^{2}\left(0, T ; \mathrm{L}^{2}(\Omega)\right)$ and $Z_{\varepsilon}^{0} \in \mathrm{L}^{2}(\Omega)(i=1,2)$, satisfy

$$
\left\{\begin{array}{l}
\text { (i) } \quad g_{i \varepsilon} \rightarrow g_{i} \quad \text { strongly in } \mathrm{L}^{2}\left(0, T ; \mathrm{L}^{2}(\Omega)\right) \\
\text { (ii) } Z_{\varepsilon}^{0} \rightarrow Z^{0} \quad \text { strongly in } \mathrm{L}^{2}(\Omega)
\end{array}\right.
$$

for some $Z^{0} \in \mathrm{L}^{2}(\Omega)$. Then

$$
\left\{\begin{array}{l}
\text { (i) } \widetilde{z_{1 \varepsilon}}+\widetilde{z_{2 \varepsilon}}=z_{\varepsilon} \rightarrow z_{1} \quad \text { in } \mathrm{C}^{0}\left([0, T] ; \mathrm{L}^{2}(\Omega)\right) \\
\text { (ii) } \lim _{\varepsilon \rightarrow 0}\left\|\nabla z_{1 \varepsilon}-C^{\varepsilon} \nabla z_{1}\right\|_{\mathrm{L}^{2}\left(0, T ;\left[\mathrm{L}^{1}\left(\Omega_{1 \varepsilon}\right)\right]^{n}\right)}=0 \\
\text { (iii) } \lim _{\varepsilon \rightarrow 0}\left\|\nabla z_{2 \varepsilon}\right\|_{\mathrm{L}^{2}\left(0, T ;\left[\mathrm{L}^{2}\left(\Omega_{2 \varepsilon}\right)\right]^{n}\right)}=0
\end{array}\right.
$$


Case $\gamma=1$ : Suppose that for $Z_{\varepsilon}^{0} \in \mathrm{L}^{2}(\Omega)$ and $g_{i \varepsilon} \in \mathrm{L}^{2}\left(0, T ; \mathrm{L}^{2}(\Omega)\right), i=1,2$ one has (2.13)(i) and

$$
\left\{\begin{array}{l}
\text { (i) } g_{i \varepsilon} \rightarrow g_{i} \quad \text { strongly in } \mathrm{L}^{2}\left(0, T ; \mathrm{L}^{2}(\Omega)\right), \\
\text { (ii) }\left\|Z_{\varepsilon}^{0}\right\|_{\mathrm{L}^{2}\left(\Omega_{1 \varepsilon}\right)}^{2}+\left\|Z_{\varepsilon}^{0}\right\|_{\mathrm{L}^{2}\left(\Omega_{2 \varepsilon}\right)}^{2} \rightarrow \theta_{1}\left\|Z_{1}^{0}\right\|_{\mathrm{L}^{2}(\Omega)}^{2}+\theta_{2}\left\|Z_{2}^{0}\right\|_{\mathrm{L}^{2}(\Omega)}^{2} .
\end{array}\right.
$$

Assuming that $\Gamma$ is of class $\mathcal{C}^{2}$, the following corrector results for the case $\gamma=1$ hold true:

$$
\left\{\begin{array}{l}
\text { (i) } \lim _{\varepsilon \rightarrow 0}\left\|z_{1 \varepsilon}-z_{1}\right\|_{\mathrm{C}^{0}\left(0, T ; \mathrm{L}^{2}\left(\Omega_{1 \varepsilon}\right)\right)}=0, \\
\text { (ii) } \lim _{\varepsilon \rightarrow 0}\left\|z_{2 \varepsilon}-\theta_{2}^{-1} z_{2}\right\|_{\mathrm{C}^{0}\left(0, T ; \mathrm{L}^{2}\left(\Omega_{2 \varepsilon}\right)\right)}=0, \\
\text { (iii) } \lim _{\varepsilon \rightarrow 0}\left\|\nabla z_{1 \varepsilon}-C^{\varepsilon} \nabla z_{1}\right\|_{\mathrm{L}^{2}\left(0, T ;\left[\mathrm{L}^{1}\left(\Omega_{1 \varepsilon}\right)\right]^{n}\right)}=0, \\
\text { (iv) } \lim _{\varepsilon \rightarrow 0}\left\|\nabla z_{2 \varepsilon}\right\|_{\mathrm{L}^{2}\left(0, T ;\left[\mathrm{L}^{2}\left(\Omega_{2 \varepsilon}\right)\right]^{n}\right)}=0 .
\end{array}\right.
$$

Remark 2.6. In particular, (2.23)(i) holds if for $i=1,2, g_{i \varepsilon}=\left.g_{\varepsilon}\right|_{\Omega_{i \varepsilon}}$ and $g_{\varepsilon} \rightarrow g$ strongly in $\mathrm{L}^{2}\left(0, T ; \mathrm{L}^{2}(\Omega)\right)$. On the other hand, it was shown in [6] that assumptions (2.13)(i) and (2.25)(ii) hold if for $i=1,2$, one has $\chi_{\Omega_{i \varepsilon}} Z_{\varepsilon}^{0}=\left.Z_{i \varepsilon}^{0}\right|_{\Omega_{i \varepsilon}}$ for some $Z_{i \varepsilon}^{0} \in \mathrm{L}^{2}(\Omega)$ such that $Z_{i \varepsilon}^{0} \rightarrow Z_{i}^{0}$ strongly in $\mathrm{L}^{2}(\Omega)$.

\section{Statement of the main Results}

In this section, we give the main results of this paper. In Section 3.1, we state the existence of an approximate control for the $\varepsilon$-problem (2.2) as well as for the corresponding homogenized problems for cases $-1<\gamma<1$ and $\gamma=1$. In Section 3.2, the main convergence result of the paper is given. The statement reveals that the control of the $\varepsilon$-problem and its corresponding solution converge (as $\varepsilon \rightarrow 0$ ) respectively to the control and to the solution of the homogenized problem.

\subsection{Controllability of the $\varepsilon$-problems and the homogenized problems}

To prove the existence of a control of the $\varepsilon$-problem, for a given $w_{\varepsilon} \in \mathrm{L}^{2}(\Omega)$ and for any $\varphi^{0} \in \mathrm{L}^{2}(\Omega)$, we define the functional $J_{\varepsilon}$ on $\mathrm{L}^{2}(\Omega)$ by

$$
\begin{aligned}
J_{\varepsilon}\left(\varphi^{0}\right)= & \frac{1}{2}\left(\int_{0}^{T} \int_{\omega_{1 \varepsilon}}\left|\varphi_{1 \varepsilon}\right|^{2} \mathrm{~d} x \mathrm{~d} t+\int_{0}^{T} \int_{\omega_{2 \varepsilon}}\left|\varphi_{2 \varepsilon}\right|^{2} \mathrm{~d} x \mathrm{~d} t\right)+\delta_{1}\left\|\varphi^{0}\right\|_{\mathrm{L}^{2}\left(\Omega_{1 \varepsilon}\right)} \\
& +\delta_{2}\left\|\varphi^{0}\right\|_{\mathrm{L}^{2}\left(\Omega_{2 \varepsilon}\right)}-\int_{\Omega_{1 \varepsilon}}\left(w_{\varepsilon}-v_{1 \varepsilon}(T)\right) \varphi^{0} \mathrm{~d} x-\int_{\Omega_{2 \varepsilon}}\left(w_{\varepsilon}-v_{2 \varepsilon}(T)\right) \varphi^{0} \mathrm{~d} x,
\end{aligned}
$$

where $\varphi_{\varepsilon}=\left(\varphi_{1 \varepsilon}, \varphi_{2 \varepsilon}\right)$ is the solution of the transposed problem of system (2.2) given by

$$
\begin{cases}-\varphi_{i \varepsilon}{ }^{\prime}-\operatorname{div}\left(A^{\varepsilon} \nabla \varphi_{i \varepsilon}\right)=0 & \text { in } \Omega_{i \varepsilon} \times(0, T), i=1,2, \\ A^{\varepsilon} \nabla \varphi_{1 \varepsilon} \cdot n_{1 \varepsilon}=-A^{\varepsilon} \nabla \varphi_{2 \varepsilon} \cdot n_{2 \varepsilon} & \text { on } \Gamma_{\varepsilon} \times(0, T), \\ A^{\varepsilon} \nabla \varphi_{1 \varepsilon} \cdot n_{1 \varepsilon}=-\varepsilon^{\gamma} h^{\varepsilon}\left(\varphi_{1 \varepsilon}-\varphi_{2 \varepsilon}\right) & \text { on } \Gamma_{\varepsilon} \times(0, T), \\ \varphi_{\varepsilon}=0 & \text { on } \partial \Omega \times(0, T), \\ \varphi_{i \varepsilon}(x, T)=\left.\varphi^{0}\right|_{\Omega_{i \varepsilon}} & \text { in } \Omega_{i \varepsilon}, i=1,2,\end{cases}
$$

and $v_{\varepsilon}=\left(v_{1 \varepsilon}, v_{2 \varepsilon}\right)$ is the solution of the auxiliary problem

$$
\begin{cases}v_{i \varepsilon}{ }^{\prime}-\operatorname{div}\left(A^{\varepsilon} \nabla v_{i \varepsilon}\right)=0 & \text { in } \Omega_{i \varepsilon} \times(0, T), i=1,2, \\ A^{\varepsilon} \nabla v_{1 \varepsilon} \cdot n_{1 \varepsilon}=-A^{\varepsilon} \nabla v_{2 \varepsilon} \cdot n_{2 \varepsilon} & \text { on } \Gamma_{\varepsilon} \times(0, T), \\ A^{\varepsilon} \nabla v_{1 \varepsilon} \cdot n_{1 \varepsilon}=-\varepsilon^{\gamma} h^{\varepsilon}\left(v_{1 \varepsilon}-v_{2 \varepsilon}\right) & \text { on } \Gamma_{\varepsilon} \times(0, T), \\ v_{\varepsilon}=0 & \text { on } \partial \Omega \times(0, T), \\ v_{i \varepsilon}(x, 0)=\left.U_{\varepsilon}^{0}\right|_{\Omega_{i \varepsilon}} & \text { in } \Omega_{i \varepsilon}, i=1,2,\end{cases}
$$

where $n_{i \varepsilon}$ is the unitary outward normal to $\Omega_{i \varepsilon}(i=1,2)$ and $U_{\varepsilon}^{0} \in \mathrm{L}^{2}(\Omega)$. 
Remark 3.1. Since the two components are disjoint, $J_{\varepsilon}\left(\varphi^{0}\right)$ given by (3.1) can be written as

$$
J_{\varepsilon}\left(\varphi^{0}\right)=\frac{1}{2} \int_{0}^{T} \int_{\omega}\left|\varphi_{\varepsilon}\right|^{2} \mathrm{~d} x \mathrm{~d} t+\delta_{1}\left\|\varphi^{0}\right\|_{\mathrm{L}^{2}\left(\Omega_{1 \varepsilon}\right)}+\delta_{2}\left\|\varphi^{0}\right\|_{\mathrm{L}^{2}\left(\Omega_{2 \varepsilon}\right)}-\int_{\Omega}\left(w_{\varepsilon}-v_{\varepsilon}(T)\right) \varphi^{0} \mathrm{~d} x
$$

Due to the different homogenization results recalled in Section 2 for the two cases $-1<\gamma<1$ and $\gamma=1$, in the sequel we mainly use the expression given by (3.4) for the case $-1<\gamma<1$ and that from (3.1) for $\gamma=1$. Indeed, in the last case one needs to handle the integral over each component.

In the following theorem, we show that for a fixed $\varepsilon$, problem (2.2) is approximately controllable in time $T$.

Theorem 3.2. Let $T>0, \delta_{1}>0, \delta_{2}>0$ be given real numbers, $w_{\varepsilon}$ be given in $\mathrm{L}^{2}(\Omega)$ and $U_{\varepsilon}^{0}$ be in $\mathrm{L}^{2}(\Omega)$. Suppose $(2.4)-(2.8)$ hold. Let $\widehat{\varphi}_{\varepsilon}^{0}$ be the unique minimum point of the functional $J_{\varepsilon}$. If $\widehat{\varphi}_{\varepsilon}=\left(\widehat{\varphi}_{1 \varepsilon}, \widehat{\varphi}_{2 \varepsilon}\right)$ is the solution of (3.2) with the corresponding final data $\widehat{\varphi}_{\varepsilon}^{0}$, then the solution $u_{\varepsilon}=\left(u_{1 \varepsilon}, u_{2 \varepsilon}\right)$ of the following system:

$$
\begin{cases}u_{i \varepsilon}{ }^{\prime}-\operatorname{div}\left(A^{\varepsilon} \nabla u_{i \varepsilon}\right)=\chi_{\omega_{i \varepsilon}} \widehat{\varphi}_{i \varepsilon} & \text { in } \Omega_{i \varepsilon} \times(0, T), i=1,2, \\ A^{\varepsilon} \nabla u_{1 \varepsilon} \cdot n_{1 \varepsilon}=-A^{\varepsilon} \nabla u_{2 \varepsilon} \cdot n_{2 \varepsilon} & \text { on } \Gamma_{\varepsilon} \times(0, T), \\ A^{\varepsilon} \nabla u_{1 \varepsilon} \cdot n_{1 \varepsilon}=-\varepsilon^{\gamma} h^{\varepsilon}\left(u_{1 \varepsilon}-u_{2 \varepsilon}\right) & \text { on } \Gamma_{\varepsilon} \times(0, T), \\ u_{\varepsilon}=0 & \text { on } \partial \Omega \times(0, T), \\ u_{i \varepsilon}(x, 0)=\left.U_{\varepsilon}^{0}\right|_{\Omega_{i \varepsilon}} & \text { in } \Omega_{i \varepsilon}, i=1,2,\end{cases}
$$

satisfies the following estimate:

$$
\left\|u_{i \varepsilon}(T)-w_{\varepsilon}\right\|_{L^{2}\left(\Omega_{i \varepsilon}\right)} \leq \delta_{i}, i=1,2 .
$$

This theorem will be proved in Section 5. Our aim being to determine whether the approximate control (found in Thm. 3.2) and the corresponding solution converge (as $\varepsilon \rightarrow 0$ ) respectively to the control and to the solution of the homogenized problem, we will separate the cases $-1<\gamma<1$ and $\gamma=1$ because of different homogenized problems (see $[6,10])$.

To to that, let us describe first the control of the homogenized problem for the two cases $-1<\gamma<1$ and $\gamma=1$. Let us start with the case where $-1<\gamma<1$, for which we have

Theorem 3.3. Under the notations of Section 2, let $T>0, \delta_{1}>0, \delta_{2}>0$ be given real numbers, $w$ be given in $\mathrm{L}^{2}(\Omega)$ and $U^{0} \in \mathrm{L}^{2}(\Omega)$. Denote $v$ the solution of the problem

$$
\begin{cases}v^{\prime}-\operatorname{div}\left(A^{0} \nabla v\right)=0 & \text { in } \Omega \times(0, T), \\ v=0 & \text { on } \partial \Omega \times(0, T), \\ v(x, 0)=U^{0} & \text { in } \Omega .\end{cases}
$$

For a given $w \in \mathrm{L}^{2}(\Omega)$, we define the functional $J_{0}$ on $\left[\mathrm{L}^{2}(\Omega)\right]^{2}$ by

$$
J_{0}\left(\Phi^{0}, \Psi^{0}\right)=\frac{1}{2} \int_{0}^{T} \int_{\omega}|\varphi|^{2} \mathrm{~d} x \mathrm{~d} t+\delta_{1} \sqrt{\theta_{1}}\left\|\Phi^{0}\right\|_{\mathrm{L}^{2}(\Omega)}+\delta_{2} \sqrt{\theta_{2}}\left\|\Psi^{0}\right\|_{\mathrm{L}^{2}(\Omega)}-\int_{\Omega}(w-v(T))\left(\theta_{1} \Phi^{0}+\theta_{2} \Psi^{0}\right) \mathrm{d} x,
$$

where $\varphi$ is the solution of the following homogeneous transposed problem:

$$
\begin{cases}-\varphi^{\prime}-\operatorname{div}\left(A^{0} \nabla \varphi\right)=0 & \text { in } \Omega \times(0, T), \\ \varphi=0 & \text { on } \partial \Omega \times(0, T), \\ \varphi(x, T)=\theta_{1} \Phi^{0}+\theta_{2} \Psi^{0} & \text { in } \Omega .\end{cases}
$$

Let $\left(\widehat{\Phi}^{0}, \widehat{\Psi}^{0}\right)$ be the unique minimum point of the functional $J_{0}$ and $\widehat{\varphi}$ the solution of (3.9) with final data $\theta_{1} \widehat{\Phi}^{0}+\theta_{2} \widehat{\Psi}^{0}$. Then if $u_{1}$ is the solution of

$$
\begin{cases}u_{1}{ }^{\prime}-\operatorname{div}\left(A^{0} \nabla u_{1}\right)=\chi_{\omega} \widehat{\varphi} & \text { in } \Omega \times(0, T), \\ u_{1}=0 & \text { on } \partial \Omega \times(0, T), \\ u_{1}(x, 0)=U^{0} & \text { in } \Omega,\end{cases}
$$


we have the following approximate controllability:

$$
\left\|u_{1}(x, T)-w\right\|_{\mathrm{L}^{2}(\Omega)} \leq \delta_{1} \sqrt{\theta_{1}}+\delta_{2} \sqrt{\theta_{2}} .
$$

This theorem is proved in Section 4. For the case $\gamma=1$, which is more interesting since the limit problem is a coupled system, we state the following result, also proved in Section 4.

Theorem 3.4. Under the notations of Section 2 , let $T>0, \delta_{1}>0, \delta_{2}>0$ be given real numbers, $w$ be given in $\mathrm{L}^{2}(\Omega)$ and $U_{1}^{0}$ and $U_{2}^{0}$ be in $\mathrm{L}^{2}(\Omega)$. Let $\left(v_{1}, v_{2}\right)$ be a solution of the problem

$$
\begin{cases}\theta_{1} v_{1}{ }^{\prime}-\operatorname{div}\left(A^{0} \nabla v_{1}\right)+c_{h}\left(\theta_{2} v_{1}-v_{2}\right)=0 & \text { in } \Omega \times(0, T), \\ v_{2}{ }^{\prime}-c_{h}\left(\theta_{2} v_{1}-v_{2}\right)=0 & \text { in } \Omega \times(0, T), \\ v_{1}=0 & \text { on } \partial \Omega \times(0, T), \\ v_{1}(x, 0)=U_{1}^{0}, \quad v_{2}(x, 0)=\theta_{2} U_{2}^{0} & \text { in } \Omega .\end{cases}
$$

For a given $w \in \mathrm{L}^{2}(\Omega)$, we define the functional $J_{0}$ on $\mathrm{L}^{2}(\Omega)$ by

$$
\begin{aligned}
J_{0}\left(\Phi^{0}, \Psi^{0}\right)= & \frac{1}{2} \theta_{1} \int_{0}^{T} \int_{\omega}\left|\varphi_{1}\right|^{2} \mathrm{~d} x \mathrm{~d} t+\frac{1}{2} \theta_{2}^{-1} \int_{0}^{T} \int_{\omega}\left|\varphi_{2}\right|^{2} \mathrm{~d} x \mathrm{~d} t+\delta_{1} \sqrt{\theta_{1}}\left\|\Phi^{0}\right\|_{\mathrm{L}^{2}(\Omega)}+\delta_{2} \sqrt{\theta_{2}}\left\|\Psi^{0}\right\|_{\mathrm{L}^{2}(\Omega)} \\
& -\theta_{1} \int_{\Omega}\left(w-v_{1}(T)\right) \Phi^{0} \mathrm{~d} x-\theta_{2} \int_{\Omega}\left(w-\theta_{2}^{-1} v_{2}(T)\right) \Psi^{0} \mathrm{~d} x,
\end{aligned}
$$

where $\left(\varphi_{1}, \varphi_{2}\right)$ is the solution of the following homogeneous transposed problem:

$$
\begin{cases}-\theta_{1} \varphi_{1}^{\prime}-\operatorname{div}\left(A^{0} \nabla \varphi_{1}\right)+c_{h}\left(\theta_{2} \varphi_{1}-\varphi_{2}\right)=0 & \text { in } \Omega \times(0, T), \\ -\varphi_{2}^{\prime}-c_{h}\left(\theta_{2} \varphi_{1}-\varphi_{2}\right)=0 & \text { in } \Omega \times(0, T), \\ \varphi_{1}=0 & \text { on } \partial \Omega \times(0, T), \\ \varphi_{1}(x, T)=\Phi^{0}, \quad \varphi_{2}(x, T)=\theta_{2} \Psi^{0} & \text { in } \Omega .\end{cases}
$$

Let $\left(\widehat{\Phi}^{0}, \widehat{\Psi}^{0}\right)$ be the unique minimum point of the functional $J_{0}$ and $\left(\widehat{\varphi}_{1}, \widehat{\varphi}_{2}\right)$ the solution of (3.14) with final data $\left(\widehat{\Phi}^{0}, \theta_{2} \widehat{\Psi}^{0}\right)$. Then if $\left(u_{1}, u_{2}\right)$ is the solution of

$$
\begin{cases}\theta_{1} u_{1}{ }^{\prime}-\operatorname{div}\left(A^{0} \nabla u_{1}\right)+c_{h}\left(\theta_{2} u_{1}-u_{2}\right)=\chi_{\omega} \theta_{1} \widehat{\varphi}_{1} & \text { in } \Omega \times(0, T), \\ u_{2}{ }^{\prime}-c_{h}\left(\theta_{2} u_{1}-u_{2}\right)=\chi_{\omega} \widehat{\varphi}_{2} & \text { in } \Omega \times(0, T), \\ u_{1}=0 & \text { on } \partial \Omega \times(0, T), \\ u_{1}(x, 0)=U_{1}^{0}, \quad u_{2}(x, 0)=\theta_{2} U_{2}^{0} & \text { in } \Omega,\end{cases}
$$

we have the following approximate controllability:

$$
\left\|\theta_{1} u_{1}(x, T)+u_{2}(x, T)-w\right\|_{L^{2}(\Omega)} \leq \delta_{1} \sqrt{\theta_{1}}+\delta_{2} \sqrt{\theta_{2}} .
$$

\subsection{Limit behavior of the approximate controllability problem}

The following theorem gives a positive answer to the second question posed in Section 2. This is the main result of the study.

Theorem 3.5. Suppose $T, \delta_{1}, \delta_{2}>0$. Let $U_{\varepsilon}^{0} \in \mathrm{L}^{2}(\Omega)$. Further, assume that $(2.4)-(2.8)$ hold and let $u_{\varepsilon}=$ $\left(u_{1 \varepsilon}, u_{2 \varepsilon}\right)$ the solution of $(3.5), \widehat{\varphi}_{\varepsilon}=\left(\widehat{\varphi}_{1 \varepsilon}, \widehat{\varphi}_{2 \varepsilon}\right)$ being the control given in Theorem 3.2.

Suppose $-1<\gamma<1$. Let $\left\{w_{\varepsilon}\right\}_{\varepsilon} \subset \mathrm{L}^{2}(\Omega)$ and $\left\{U_{\varepsilon}^{0}\right\}_{\varepsilon} \subset \mathrm{L}^{2}(\Omega)$ satisfy the following assumptions:

$$
\begin{cases}(\mathrm{i}) \quad U_{\varepsilon}^{0} \rightarrow U^{0} & \text { strongly in } \mathrm{L}^{2}(\Omega), \\ \text { (ii) } \quad w_{\varepsilon} \rightarrow w & \text { strongly in } \mathrm{L}^{2}(\Omega),\end{cases}
$$


for some $U^{0}$ and $w$ in $\mathrm{L}^{2}(\Omega)$. Then, as $\varepsilon \rightarrow 0$, we have the following convergences:

$$
\begin{cases}\text { (i) } \widehat{\varphi}_{\varepsilon} \rightarrow \widehat{\varphi} & \text { strongly in } \mathrm{L}^{2}\left(0, T ; \mathrm{L}^{2}(\Omega)\right), \\ \text { (ii) }\left(\chi_{\Omega_{1 \varepsilon}} \widehat{\varphi}_{\varepsilon}^{0}, \chi_{\Omega_{2 \varepsilon}} \widehat{\varphi}_{\varepsilon}^{0}\right) \rightarrow\left(\theta_{1} \widehat{\Phi}^{0}, \theta_{2} \widehat{\Psi}^{0}\right) & \text { weakly in }\left[\mathrm{L}^{2}(\Omega)\right]^{2},\end{cases}
$$

where $\widehat{\varphi}$ is the solution of (3.9) with final data $\theta_{1} \widehat{\Phi}^{0}+\theta_{2} \widehat{\Psi}^{0}$ and $\left(\widehat{\Phi}^{0}, \widehat{\Psi}^{0}\right)$ is the unique minimum point of the functional $J_{0}$ defined by (3.8). Moreover,

$$
\begin{cases}\text { (i) } \quad P_{1}^{\varepsilon} u_{1 \varepsilon} \rightarrow u_{1} & \text { weakly in } \mathrm{L}^{2}\left(0, T ; \mathrm{H}_{0}^{1}(\Omega)\right) \\ \text { (ii) } u_{\varepsilon} \rightarrow u_{1} & \text { strongly in } \mathrm{C}^{0}\left([0, T] ; \mathrm{L}^{2}(\Omega)\right) \\ \text { (iii) } \widetilde{u_{1 \varepsilon}} \rightarrow \theta_{1} u_{1} & \text { weakly* in } \mathrm{L}^{\infty}\left(0, T ; \mathrm{L}^{2}(\Omega)\right)\end{cases}
$$

where $u_{1}$ is the solution of

$$
\begin{cases}u_{1}{ }^{\prime}-\operatorname{div}\left(A^{0} \nabla u_{1}\right)=\chi_{\omega} \widehat{\varphi} & \text { in } \Omega \times(0, T) \\ u_{1}=0 & \text { on } \partial \Omega \times(0, T) \\ u_{1}(x, 0)=U^{0} & \text { in } \Omega .\end{cases}
$$

The function $\widehat{\varphi}$ is an approximate control for the homogenized problem (3.20) corresponding to $w$ and the constants $\delta_{1}$ and $\delta_{2}$, that is

$$
\left\|u_{1}(x, T)-w\right\|_{\mathrm{L}^{2}(\Omega)} \leq \delta_{1} \sqrt{\theta_{1}}+\delta_{2} \sqrt{\theta_{2}}
$$

Finally, one has the following convergence:

$$
\delta_{1}\left\|\widehat{\varphi}_{\varepsilon}^{0}\right\|_{L^{2}\left(\Omega_{1 \varepsilon}\right)}+\delta_{2}\left\|\widehat{\varphi}_{\varepsilon}^{0}\right\|_{L^{2}\left(\Omega_{2 \varepsilon}\right)} \rightarrow \delta_{1} \sqrt{\theta_{1}}\left\|\widehat{\Phi}^{0}\right\|_{L^{2}(\Omega)}+\delta_{2} \sqrt{\theta_{2}}\left\|\widehat{\Psi}^{0}\right\|_{L^{2}(\Omega)}
$$

so that in particular, if $\delta_{1}=\delta_{2}$ then

$$
\left\|\widehat{\varphi}_{\varepsilon}^{0}\right\|_{L^{2}(\Omega)} \rightarrow \sqrt{\theta_{1}}\left\|\widehat{\Phi}^{0}\right\|_{L^{2}(\Omega)}+\sqrt{\theta_{2}}\left\|\widehat{\Psi}^{0}\right\|_{L^{2}(\Omega)} .
$$

Now, let $\gamma=1$ and assume that $\Gamma$ is of class $\mathcal{C}^{2}$. For $\left\{w_{\varepsilon}\right\}_{\varepsilon} \subset \mathrm{L}^{2}(\Omega)$ and $\left\{U_{\varepsilon}^{0}\right\}_{\varepsilon} \subset \mathrm{L}^{2}(\Omega)$, we suppose that for some $U_{i}^{0}, i=1,2$ and $w$ in $\mathrm{L}^{2}(\Omega)$, they satisfy the following assumptions:

$$
\left\{\begin{array}{l}
\left(\text { i) } \quad \chi_{\Omega_{i \varepsilon}} U_{\varepsilon}^{0} \rightarrow \theta_{i} U_{i}^{0} \quad \text { weakly in } \mathrm{L}^{2}(\Omega),\right. \\
\text { (ii) }\left\|U_{\varepsilon}^{0}\right\|_{\mathrm{L}^{2}\left(\Omega_{1 \varepsilon}\right)}^{2}+\left\|U_{\varepsilon}^{0}\right\|_{\mathrm{L}^{2}\left(\Omega_{2 \varepsilon}\right)}^{2} \rightarrow \theta_{1}\left\|U_{1}^{0}\right\|_{\mathrm{L}^{2}(\Omega)}^{2}+\theta_{2}\left\|U_{2}^{0}\right\|_{\mathrm{L}^{2}(\Omega)}^{2}, \\
\text { (iii) } w_{\varepsilon} \rightarrow w \text { strongly in } \mathrm{L}^{2}(\Omega) .
\end{array}\right.
$$

Then as $\varepsilon \rightarrow 0$, one has

$$
\begin{cases}(\mathrm{i}) \quad \chi_{\omega_{1 \varepsilon}} \widetilde{\widehat{\varphi}_{1 \varepsilon}} \rightarrow \chi_{\omega} \theta_{1} \widehat{\varphi}_{1} & \text { weakly in } \mathrm{L}^{2}\left(0, T ; \mathrm{L}^{2}(\Omega)\right), \\ \text { (ii) } \chi_{\omega_{2 \varepsilon}}{\widehat{\widehat{\varphi}_{2 \varepsilon}}}_{\widehat{\varphi}} \chi_{\omega} \widehat{\varphi}_{2} & \text { weakly in } \mathrm{L}^{2}\left(0, T ; \mathrm{L}^{2}(\Omega)\right), \\ \text { (iii) }\left(\chi_{\Omega_{1 \varepsilon}} \widehat{\varphi}_{\varepsilon}^{0}, \chi_{\Omega_{2 \varepsilon}} \widehat{\varphi}_{\varepsilon}^{0}\right) \rightarrow\left(\theta_{1} \widehat{\Phi}^{0}, \theta_{2} \widehat{\Psi}^{0}\right) & \text { weakly in }\left[\mathrm{L}^{2}(\Omega)\right]^{2}\end{cases}
$$


where $\left(\widehat{\varphi}_{1}, \widehat{\varphi}_{2}\right)$ is the solution of (3.14) with final data $\left(\widehat{\Phi}^{0}, \theta_{2} \widehat{\Psi}^{0}\right)$ and $\left(\widehat{\Phi}^{0}, \widehat{\Psi}^{0}\right)$ is the unique minimum point of the functional $J_{0}$ defined by (3.13). Moreover,

$$
\begin{cases}\text { (i) } \quad P_{1}^{\varepsilon} u_{1 \varepsilon} \rightarrow u_{1} & \text { weakly in } \mathrm{L}^{2}\left(0, T ; \mathrm{H}_{0}^{1}(\Omega)\right), \\ \text { (ii) } \widetilde{u_{1 \varepsilon}} \rightarrow \theta_{1} u_{1} & \text { weakly* in } \mathrm{L}^{\infty}\left(0, T ; \mathrm{L}^{2}(\Omega)\right), \\ \text { (iii) } \widetilde{u_{2 \varepsilon}} \rightarrow u_{2} & \text { weakly* in } \mathrm{L}^{\infty}\left(0, T ; \mathrm{L}^{2}(\Omega)\right),\end{cases}
$$

where the couple $\left(u_{1}, u_{2}\right)$ satisfies

$$
\begin{cases}\theta_{1} u_{1}{ }^{\prime}-\operatorname{div}\left(A^{0} \nabla u_{1}\right)+c_{h}\left(\theta_{2} u_{1}-u_{2}\right)=\theta_{1} \chi_{\omega} \widehat{\varphi}_{1} & \text { in } \Omega \times(0, T), \\ u_{2}{ }^{\prime}-c_{h}\left(\theta_{2} u_{1}-u_{2}\right)=\chi_{\omega} \widehat{\varphi}_{2} & \text { in } \Omega \times(0, T), \\ u_{1}=0 & \text { on } \partial \Omega \times(0, T), \\ u_{1}(x, 0)=U_{1}^{0}, \quad u_{2}(x, 0)=\theta_{2} U_{2}^{0} & \text { in } \Omega .\end{cases}
$$

The couple $\left(\widehat{\varphi}_{1}, \widehat{\varphi}_{2}\right)$ is an approximate control for the homogenized problem (3.27) corresponding to $w$ and the constants $\delta_{1}$ and $\delta_{2}$, that is

$$
\left\|\theta_{1} u_{1}(x, T)+u_{2}(x, T)-w\right\|_{\mathrm{L}^{2}(\Omega)} \leq \delta_{1} \sqrt{\theta_{1}}+\delta_{2} \sqrt{\theta_{2}} .
$$

Remark 3.6. For comments about assumption (3.24), we refer to Remark 2.6. Let us also mention the case of the controllability of the parabolic equations in the perforated domain studied in [7], wherein a factor $\frac{1}{\sqrt{\theta}}$ appears in the controllability condition for the homogenized problem (3.21).

Theorem 3.5 will be proved by using several results in the asymptotic behavior of the control for the $\varepsilon$-problem. To do this, we require our data to satisfy (3.17) and (3.24) depending on $\gamma$. We give immediate consequences of these assumptions as follow.

Remark 3.7. Let $w_{\varepsilon}$ and $w$ be in $\mathrm{L}^{2}(\Omega)$. If $-1<\gamma<1$ and Assumption (3.17) holds, using Theorem 2.5 applied to problem (3.3) we deduce that

$$
w_{\varepsilon}-v_{\varepsilon}(T) \rightarrow w-v(T) \quad \text { strongly in } \mathrm{L}^{2}(\Omega),
$$

where $\left(v_{1 \varepsilon}, v_{2 \varepsilon}\right)$ and $v$ are the solutions of (3.3) and (3.7), respectively.

Suppose now that $\gamma=1$ and Assumption (3.24) holds. Then, from (3.24)(i) and (ii) and Theorem 2.5 applied to problem (3.3) we get

$$
\left\|v_{1 \varepsilon}(T)-v_{1}(T)\right\|_{\mathrm{L}^{2}\left(\Omega_{1 \varepsilon}\right)} \rightarrow 0 \quad \text { and } \quad\left\|v_{2 \varepsilon}(T)-\theta_{2}^{-1} v_{2}(T)\right\|_{\mathrm{L}^{2}\left(\Omega_{2 \varepsilon}\right)} \rightarrow 0,
$$

where $v_{\varepsilon}=\left(v_{1 \varepsilon}, v_{2 \varepsilon}\right)$ and $\left(v_{1}, v_{2}\right)$ are the solutions of (3.3) and (3.12), respectively. This together with (3.24) (iii), yields

$$
\left\|\left(w_{\varepsilon}-v_{1 \varepsilon}(T)\right)-\left(w-v_{1}(T)\right)\right\|_{\mathrm{L}^{2}\left(\Omega_{1 \varepsilon}\right)} \rightarrow 0 \quad \text { and } \quad\left\|\left(w_{\varepsilon}-v_{2 \varepsilon}(T)\right)-\left(w-\theta_{2}^{-1} v_{2}(T)\right)\right\|_{\mathrm{L}^{2}\left(\Omega_{2 \varepsilon}\right)} \rightarrow 0 .
$$

\section{Proof of the control of the homogenized problems}

In this section, we prove the approximate controllability of the homogenized problems stated in Theorems 3.3 and 3.4 for the two cases of the parameter, respectively. To do that, we use the variational approach to derive the approximate controls of the homogenized problems, which is constructive compared to the Hahn-Banach's Theorem. This is also the technique used to study the approximate controllability of semilinear heat equation in [9], the linear parabolic equations with rapidly oscillating coefficients in a fixed domain in [13] and of the linear parabolic equations in perforated domains in [7]. 
Here, we need to construct two different functionals. The first one is adapted to the case $-1<\gamma<1$, where we only have one partial differential equation in the homogenized problem. The second one is related to that of $\gamma=1$, where in the homogenized problem we have a coupled system of a partial and an ordinary differential equation, which renders this case more difficult.

Proof of Theorem 3.3. By standard arguments one can prove that the functional $J_{0}$ given in (3.8) is continuous and strictly convex. Let us prove, in the spirit of $[9,13]$, that for any sequence $\left\{\left(\Phi_{n}^{0}, \Psi_{n}^{0}\right)\right\}$ in $\left[\mathrm{L}^{2}(\Omega)\right]^{2}$ such that $\left\|\left(\Phi_{n}^{0}, \Psi_{n}^{0}\right)\right\|_{\left[\mathrm{L}^{2}(\Omega)\right]^{2}} \rightarrow \infty$, one has

$$
\liminf _{n \rightarrow \infty} \frac{J_{0}\left(\Phi_{n}^{0}, \Psi_{n}^{0}\right)}{\left\|\left(\Phi_{n}^{0}, \Psi_{n}^{0}\right)\right\|_{\left[\mathrm{L}^{2}(\Omega)\right]^{2}}} \geq \min \left\{\delta_{1} \sqrt{\theta_{1}}, \delta_{2} \sqrt{\theta_{2}}\right\}
$$

We set

$$
\left(\bar{\Phi}_{n}^{0}, \bar{\Psi}_{n}^{0}\right)=\left(\frac{\Phi_{n}^{0}}{\left\|\left(\Phi_{n}^{0}, \Psi_{n}^{0}\right)\right\|_{\left[\mathrm{L}^{2}(\Omega)\right]^{2}}}, \frac{\Psi_{n}^{0}}{\left\|\left(\Phi_{n}^{0}, \Psi_{n}^{0}\right)\right\|_{\left[\mathrm{L}^{2}(\Omega)\right]^{2}}}\right) \quad \text { and } \quad \bar{\varphi}_{n}=\frac{\varphi_{n}}{\left\|\left(\Phi_{n}^{0}, \Psi_{n}^{0}\right)\right\|_{\left[\mathrm{L}^{2}(\Omega)\right]^{2}}},
$$

where $\varphi_{n}$ is the solution of the adjoint problem (3.9) with final data $\theta_{1} \Phi_{n}^{0}+\theta_{2} \Psi_{n}^{0}$.

Then, by the definition of the functional $J_{0}$ given in (3.8),

$$
\begin{aligned}
& \frac{J_{0}\left(\Phi_{n}^{0}, \Psi_{n}^{0}\right)}{\left\|\left(\Phi_{n}^{0}, \Psi_{n}^{0}\right)\right\|_{\left[\mathrm{L}^{2}(\Omega)\right]^{2}}}=\frac{1}{2}\left\|\left(\Phi_{n}^{0}, \Psi_{n}^{0}\right)\right\|_{\left[\mathrm{L}^{2}(\Omega)\right]^{2}} \int_{0}^{T} \int_{\omega}\left|\bar{\varphi}_{n}\right|^{2} \mathrm{~d} x \mathrm{~d} t \\
& \quad+\frac{\delta_{1} \sqrt{\theta_{1}}\left\|\Phi_{n}^{0}\right\|_{\mathrm{L}^{2}(\Omega)}+\delta_{2} \sqrt{\theta_{2}}\left\|\Psi_{n}^{0}\right\|_{\mathrm{L}^{2}(\Omega)}}{\left\|\left(\Phi_{n}^{0}, \Psi_{n}^{0}\right)\right\|_{\left[\mathrm{L}^{2}(\Omega)\right]^{2}}}-\int_{\Omega}(w-v(T))\left(\theta_{1} \bar{\Phi}_{n}^{0}+\theta_{2} \bar{\Psi}_{n}^{0}\right) \mathrm{d} x .
\end{aligned}
$$

Case 1. $\liminf _{n \rightarrow \infty} \int_{0}^{T} \int_{\omega}\left|\bar{\varphi}_{n}\right|^{2} \mathrm{~d} x \mathrm{~d} t>0$. Since $\left\|\left(\Phi_{n}^{0}, \Psi_{n}^{0}\right)\right\|_{\left[\mathrm{L}^{2}(\Omega)\right]^{2}} \rightarrow \infty$, the first term in the right-hand side of (4.2) goes to infinity. On the other hand, the second term is smaller than $\delta_{1} \sqrt{\theta_{1}}+\delta_{2} \sqrt{\theta_{2}}$. Furthermore, by the Hölder inequality, triangle inequality is easily seen so that the third one is bounded, which gives (4.1).

Case 2. $\liminf _{n \rightarrow \infty} \int_{0}^{T} \int_{\omega}\left|\bar{\varphi}_{n}\right|^{2} \mathrm{~d} x \mathrm{~d} t=0$. Since $\mathrm{L}^{2}(\Omega)$ is reflexive and $\bar{\Phi}_{n}^{0}, \bar{\Psi}_{n}^{0}$ are bounded in $\mathrm{L}^{2}(\Omega)$, we have (up to a subsequence) that

$$
\theta_{1} \bar{\Phi}_{n}^{0}+\theta_{2} \bar{\Psi}_{n}^{0} \rightarrow \sigma^{0} \quad \text { weakly in } \mathrm{L}^{2}(\Omega),
$$

for some $\sigma^{0}$ in $\mathrm{L}^{2}(\Omega)$. By linearity, passing to the limit in (3.9) (written with final data $\theta_{1} \bar{\Phi}_{n}^{0}+\theta_{2} \bar{\Psi}_{n}^{0}$ ),

$$
\bar{\varphi}_{n} \rightarrow \psi \quad \text { weakly in } \mathrm{L}^{2}\left(0, T ; \mathrm{H}_{0}^{1}(\Omega)\right) \cap \mathrm{H}^{1}\left(0, T ; \mathrm{H}^{-1}(\Omega)\right),
$$

where $\psi$ is the solution of (3.9) with $\sigma^{0}$ as final data. By lower semi-continuity and the assumption done in this case, $\psi=0$ in $\omega \times(0, T)$. From the unique-continuation property due to Saut and Scheurer [12], we deduce that $\psi=0$ in $\Omega \times(0, T)$. Since $\psi$ is in $\left.\mathrm{C}^{0}\left([0, T] ; \mathrm{L}^{2}(\Omega)\right)\right)$ and satisfies (3.9), this implies using (4.3) that $\sigma^{0}=\psi(T)=0$. Consequently, the last term in (4.2) goes to zero as $n \rightarrow \infty$. Together with our assumption, this gives (4.1), since the second term is bigger than $\min \left\{\delta_{1} \sqrt{\theta_{1}}, \delta_{2} \sqrt{\theta_{2}}\right\}$. Hence, $J_{0}$ admits a unique minimum point $\left(\widehat{\Phi}^{0}, \widehat{\Psi}^{0}\right)$ in $\left[\mathrm{L}^{2}(\Omega)\right]^{2}$. Let us show that if $\widehat{\varphi}$ is the solution of (3.9) with $\theta_{1} \widehat{\Phi}^{0}+\theta_{2} \widehat{\Psi}^{0}$ as final data, then for every $\tau^{0} \in \mathrm{L}^{2}(\Omega)$ we have

$$
\left|\int_{0}^{T} \int_{\omega} \widehat{\varphi} \psi \mathrm{d} x \mathrm{~d} t-\int_{\Omega}(w-v(T)) \tau^{0} \mathrm{~d} x\right| \leq\left(\delta_{1} \sqrt{\theta_{1}}+\delta_{2} \sqrt{\theta_{2}}\right)\left\|\tau^{0}\right\|_{\mathrm{L}^{2}(\Omega)},
$$

where $\psi$ is the solution of (3.9) with $\tau^{0}=\theta_{1} \tau^{0}+\theta_{2} \tau^{0}$ as final data. 
To do that, observe that if $J_{0}$ attains its minimum at $\left(\widehat{\Phi}^{0}, \widehat{\Psi}^{0}\right)$ then for any $h \in \mathbb{R}$, we have

$$
\begin{aligned}
0 \leq & J_{0}\left(\widehat{\Phi}^{0}+h \tau^{0}, \widehat{\Psi}^{0}+h \tau^{0}\right)-J_{0}\left(\widehat{\Phi}^{0}, \widehat{\Psi}^{0}\right) \\
= & h \int_{0}^{T} \int_{\omega} \widehat{\varphi} \psi \mathrm{d} x \mathrm{~d} t+\frac{h^{2}}{2} \int_{0}^{T} \int_{\omega}|\psi|^{2} \mathrm{~d} x \mathrm{~d} t+\delta_{1} \sqrt{\theta_{1}}\left(\left\|\widehat{\Phi}^{0}+h \tau^{0}\right\|_{\mathrm{L}^{2}(\Omega)}-\left\|\widehat{\Phi}^{0}\right\|_{\mathrm{L}^{2}(\Omega)}\right) \\
& \quad+\delta_{2} \sqrt{\theta_{2}}\left(\left\|\widehat{\Psi}^{0}+h \tau^{0}\right\|_{\mathrm{L}^{2}(\Omega)}-\left\|\widehat{\Psi}^{0}\right\|_{\mathrm{L}^{2}(\Omega)}\right)-h \int_{\Omega}(w-v(T)) \tau^{0} \mathrm{~d} x .
\end{aligned}
$$

By triangle inequality, it follows that

$$
\begin{aligned}
0 \leq & \frac{h^{2}}{2} \int_{0}^{T} \int_{\omega}|\psi|^{2} \mathrm{~d} x \mathrm{~d} t+\delta_{1} \sqrt{\theta_{1}}|h|\left\|\tau^{0}\right\|_{\mathrm{L}^{2}(\Omega)}+\delta_{2} \sqrt{\theta_{2}}|h|\left\|\tau^{0}\right\|_{\mathrm{L}^{2}(\Omega)} \\
& +h\left(\int_{0}^{T} \int_{\omega} \widehat{\varphi} \psi \mathrm{d} x \mathrm{~d} t-\int_{\Omega}(w-v(T)) \tau^{0} \mathrm{~d} x\right) .
\end{aligned}
$$

Dividing by $h>0$ and passing to the limit as $h \rightarrow 0$, we get

$$
-\left(\delta_{1} \sqrt{\theta_{1}}+\delta_{2} \sqrt{\theta_{2}}\right)\left\|\tau^{0}\right\|_{\mathrm{L}^{2}(\Omega)} \leq \int_{0}^{T} \int_{\omega} \widehat{\varphi} \psi \mathrm{d} x \mathrm{~d} t-\int_{\Omega}(w-v(T)) \tau^{0} \mathrm{~d} x .
$$

Together with an analogous computation for the case when $h<0$, we have (4.4).

Now to conclude the proof let as above $\widehat{\varphi}$ be the solution of (3.9) with $\theta_{1} \widehat{\Phi}^{0}+\theta_{2} \widehat{\Psi}^{0}$ as final data, $\tau^{0}$ in $\operatorname{L}^{2}(\Omega)$ and $z$ be the solution of the following equation:

$$
\begin{cases}z^{\prime}-\operatorname{div}\left(A^{0} \nabla z\right)=\chi_{\omega} \widehat{\varphi} & \text { in } \Omega \times(0, T), \\ z=0 & \text { on } \partial \Omega \times(0, T), \\ z(x, 0)=0 & \text { in } \Omega .\end{cases}
$$

Using as test function in (4.5) the solution $\psi$ of (3.9) with $\tau^{0}$ as final data, we have

$$
\int_{0}^{T} \int_{\Omega}\left(-\psi^{\prime} z+A^{0} \nabla \psi \nabla z\right) \mathrm{d} x \mathrm{~d} t+\int_{\Omega} z(T) \psi(T) \mathrm{d} x=\int_{0}^{T} \int_{\omega} \widehat{\varphi} \psi \mathrm{d} x \mathrm{~d} t=\int_{\Omega} z(T) \tau^{0} \mathrm{~d} x .
$$

Then, in view of (4.4),

$$
\left|\int_{\Omega} z(T) \tau^{0} \mathrm{~d} x-\int_{\Omega}(w-v(T)) \tau^{0} \mathrm{~d} x\right| \leq\left(\delta_{1} \sqrt{\theta_{1}}+\delta_{2} \sqrt{\theta_{2}}\right)\left\|\tau^{0}\right\|_{\mathrm{L}^{2}(\Omega)} .
$$

This proves (3.11), since from the definition of $u_{1}$ and $v$ one has by linearity, $u_{1}(T)=z(T)+v(T)$.

Proof of Theorem 3.4. Let $w, \Phi^{0}, \Psi^{0} \in \mathrm{L}^{2}(\Omega)$ and the functional $J_{0}$ be defined as in (3.13). The proof is similar to that of Theorem 3.3 and we only point out the main differences. Again standard arguments show that the functional $J_{0}$ given in (3.8) is continuous and strictly convex. As in the previous proof, let us show inequality (4.1), here for any sequence such that $\left\|\left(\Phi_{n}^{0}, \Psi_{n}^{0}\right)\right\|_{\left[\mathrm{L}^{2}(\Omega)\right]^{2}} \rightarrow \infty$. We set

$$
\left(\bar{\Phi}_{n}^{0}, \bar{\Psi}_{n}^{0}\right)=\left(\frac{\Phi_{n}^{0}}{\left\|\left(\Phi_{n}^{0}, \Psi_{n}^{0}\right)\right\|_{\left[\mathrm{L}^{2}(\Omega)\right]^{2}}}, \frac{\Psi_{n}^{0}}{\left\|\left(\Phi_{n}^{0}, \Psi_{n}^{0}\right)\right\|_{\left[\mathrm{L}^{2}(\Omega)\right]^{2}}}\right) \quad\left(\bar{\varphi}_{1 n}, \bar{\varphi}_{2 n}\right)=\left(\frac{\varphi_{1 n}}{\left\|\left(\Phi_{n}^{0}, \Psi_{n}^{0}\right)\right\|_{\left[\mathrm{L}^{2}(\Omega)\right]^{2}}}, \frac{\varphi_{2 n}}{\left\|\left(\Phi_{n}^{0}, \Psi_{n}^{0}\right)\right\|_{\left[\mathrm{L}^{2}(\Omega)\right]^{2}}}\right),
$$

where $\left(\varphi_{1 n}, \varphi_{2 n}\right)$ is a solution of the adjoint problem (3.14) with final data $\left(\Phi_{n}^{0}, \theta_{2} \Psi_{n}^{0}\right)$. Now by (3.13),

$$
\begin{aligned}
& \frac{J_{0}\left(\Phi_{n}^{0}, \Psi_{n}^{0}\right)}{\left\|\left(\Phi_{n}^{0}, \Psi_{n}^{0}\right)\right\|_{\left[\mathrm{L}^{2}(\Omega)\right]^{2}}}=\frac{1}{2}\left\|\left(\Phi_{n}^{0}, \Psi_{n}^{0}\right)\right\|_{\left[\mathrm{L}^{2}(\Omega)\right]^{2}}\left(\theta_{1} \int_{0}^{T} \int_{\omega}\left|\bar{\varphi}_{1 n}\right|^{2} \mathrm{~d} x \mathrm{~d} t+\theta_{2}^{-1} \int_{0}^{T} \int_{\omega}\left|\bar{\varphi}_{2 n}\right|^{2} \mathrm{~d} x \mathrm{~d} t\right) \\
& +\frac{\delta_{1} \sqrt{\theta_{1}}\left\|\Phi_{n}^{0}\right\|_{\mathrm{L}^{2}(\Omega)}+\delta_{2} \sqrt{\theta_{2}}\left\|\Psi_{n}^{0}\right\|_{\mathrm{L}^{2}(\Omega)}}{\left\|\left(\Phi_{n}^{0}, \Psi_{n}^{0}\right)\right\|_{\left[\mathrm{L}^{2}(\Omega)\right]^{2}}}-\theta_{1} \int_{\Omega}\left(w-v_{1}(T)\right) \bar{\Phi}_{n}^{0} \mathrm{~d} x-\theta_{2} \int_{\Omega}\left(w-\theta_{2}^{-1} v_{2}(T)\right) \bar{\Psi}_{n}^{0} \mathrm{~d} x .
\end{aligned}
$$


Case 1. At least one of the $\liminf _{n \rightarrow \infty} \int_{0}^{T} \int_{\omega}\left|\bar{\varphi}_{i n}\right|^{2} \mathrm{~d} x \mathrm{~d} t, i=1,2$ is strictly positive. Then,

$$
\frac{1}{2}\left\|\left(\Phi_{n}^{0}, \Psi_{n}^{0}\right)\right\|_{\left[\mathrm{L}^{2}(\Omega)\right]^{2}}\left(\theta_{1} \int_{0}^{T} \int_{\omega}\left|\bar{\varphi}_{1 n}\right|^{2} \mathrm{~d} x \mathrm{~d} t+\theta_{2}^{-1} \int_{0}^{T} \int_{\omega}\left|\bar{\varphi}_{2 n}\right|^{2} \mathrm{~d} x \mathrm{~d} t\right) \rightarrow \infty .
$$

and we conclude as in the proof of the corresponding case for Theorem 3.3.

Case 2. $\liminf _{n \rightarrow \infty} \int_{0}^{T} \int_{\omega}\left|\bar{\varphi}_{1 n}\right|^{2} \mathrm{~d} x \mathrm{~d} t=\liminf _{n \rightarrow \infty} \int_{0}^{T} \int_{\omega}\left|\bar{\varphi}_{2 n}\right|^{2} \mathrm{~d} x \mathrm{~d} t=0$.

Since $\bar{\Phi}_{n}^{0}, \bar{\Psi}_{n}^{0}$ are bounded in $\mathrm{L}^{2}(\Omega)$, up to a subsequence, we have for some $\sigma^{0}$ and $\tau^{0}$ in $\mathrm{L}^{2}(\Omega)$

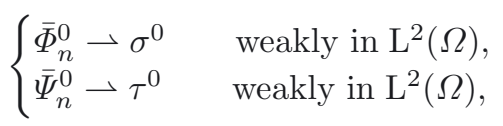

By linearity, passing to the limit in (3.14) (written with final data $\left(\bar{\Phi}_{n}^{0}, \theta_{2} \bar{\Psi}_{n}^{0}\right)$ ), one has

$$
\bar{\varphi}_{\text {in }} \rightarrow \psi_{i} \quad \text { weakly in } \mathrm{L}^{2}\left(0, T ; \mathrm{H}_{0}^{1}(\Omega)\right), \quad i=1,2,
$$

where $\left(\psi_{1}, \psi_{2}\right)$ is the solution of $(3.14)$ with $\left(\sigma^{0}, \theta_{2} \tau^{0}\right)$ as final data. By lower semi-continuity, and the assumption for this case, $\psi_{1}$ and $\psi_{2}$ are zero in $\omega \times(0, T)$ so that from Theorem 7.1 of the Appendix, $\psi_{1}, \psi_{2}$ are zero in $\Omega \times(0, T)$. Since $\left.\psi_{1}, \psi_{2} \in \mathrm{C}^{0}\left([0, T] ; \mathrm{L}^{2}(\Omega)\right)\right)$ and $\psi_{1}, \psi_{2}$ satisfy (3.14), in view of (4.7) we have

$$
\begin{cases}\bar{\Phi}_{n}^{0} \rightarrow \sigma^{0}=\psi_{1}(T)=0 & \text { weakly in } \mathrm{L}^{2}(\Omega), \\ \bar{\Psi}_{n}^{0} \rightarrow \theta_{2} \tau^{0}=\psi_{2}(T)=0 & \text { weakly in } \mathrm{L}^{2}(\Omega) .\end{cases}
$$

Therefore, the sum of the two last terms in the right-hand side of (4.6) goes to zero. This, together with the assumption for this case and arguing as in the Proof of Theorem 3.3, concludes the proof of (4.1).

Let us prove now that if $\left(\widehat{\Phi}^{0}, \widehat{\Psi}^{0}\right) \in\left[\mathrm{L}^{2}(\Omega)\right]^{2}$ is the minimum point of $J_{0}$ then, for any $\tau^{0} \in \mathrm{L}^{2}(\Omega)$,

$$
\begin{gathered}
\mid \int_{0}^{T} \int_{\omega} \theta_{1} \widehat{\varphi}_{1} \psi_{1} \mathrm{~d} x \mathrm{~d} t+\int_{0}^{T} \int_{\omega} \theta_{2}^{-1} \widehat{\varphi}_{2} \psi_{2} \mathrm{~d} x \mathrm{~d} t-\int_{\Omega}\left(w-v_{1}(T)\right) \theta_{1} \tau^{0} \mathrm{~d} x \\
-\int_{\Omega}\left(w-\theta_{2}^{-1} v_{2}(T)\right) \theta_{2} \tau^{0} \mathrm{~d} x \mid \leq\left(\delta_{1} \sqrt{\theta_{1}}+\delta_{2} \sqrt{\theta_{2}}\right)\left\|\tau^{0}\right\|_{\mathrm{L}^{2}(\Omega)},
\end{gathered}
$$

where $\left(\widehat{\varphi}_{1}, \widehat{\varphi}_{2}\right)$ is the solution of $(3.14)$ with $\left(\widehat{\Phi}^{0}, \theta_{2} \widehat{\Psi}^{0}\right)$ as final data.

To show (4.8), it must be noted that if $J_{0}$ attains its minimum at $\left(\widehat{\Phi}^{0}, \widehat{\Psi}^{0}\right)$ then we have

$$
J_{0}\left(\widehat{\Phi}^{0}, \widehat{\Psi}^{0}\right) \leq J_{0}\left(\widehat{\Phi}^{0}+h \tau^{0}, \widehat{\Psi}^{0}+h \tau^{0}\right),
$$

for any $\tau^{0} \in \mathrm{L}^{2}(\Omega)$ and $h \in \mathbb{R}$. Thus, from the definition of $J_{0}$,

$$
\begin{aligned}
0 \leq & J_{0}\left(\widehat{\Phi}^{0}+h \tau^{0}, \widehat{\Psi}^{0}+h \tau^{0}\right)-J_{0}\left(\widehat{\Phi}^{0}, \widehat{\Psi}^{0}\right)=\theta_{1} h \int_{0}^{T} \int_{\omega} \widehat{\varphi}_{1} \psi_{1} \mathrm{~d} x \mathrm{~d} t \\
& +\frac{\theta_{1} h^{2}}{2} \int_{0}^{T} \int_{\omega}\left|\psi_{1}\right|^{2} \mathrm{~d} x \mathrm{~d} t+\theta_{2}^{-1} h \int_{0}^{T} \int_{\omega} \widehat{\varphi}_{2} \psi_{2} \mathrm{~d} x \mathrm{~d} t+\frac{\theta_{2}^{-1} h^{2}}{2} \int_{0}^{T} \int_{\omega}\left|\psi_{2}\right|^{2} \mathrm{~d} x \mathrm{~d} t \\
& +\delta_{1} \sqrt{\theta_{1}}\left(\left\|\widehat{\Phi}^{0}+h \tau^{0}\right\|_{\mathrm{L}^{2}(\Omega)}-\left\|\widehat{\Phi}^{0}\right\|_{\mathrm{L}^{2}(\Omega)}\right)+\delta_{2} \sqrt{\theta_{2}}\left(\left\|\widehat{\Psi}^{0}+h \tau^{0}\right\|_{\mathrm{L}^{2}(\Omega)}-\left\|\widehat{\Psi}^{0}\right\|_{\mathrm{L}^{2}(\Omega)}\right) \\
& -h\left(\int_{\Omega}\left(w-v_{1}(T)\right) \theta_{1} \tau^{0} \mathrm{~d} x-\int_{\Omega}\left(w-\theta_{2}^{-1} v_{2}(T)\right) \theta_{2} \tau^{0} \mathrm{~d} x\right) .
\end{aligned}
$$

Arguing as in the proof for the case $\gamma<1$, we obtain (4.8). 
To complete the Proof of Theorem 3.4, let $\left(\widehat{\varphi}_{1}, \widehat{\varphi}_{2}\right)$ as above and for any $\tau^{0} \in \mathrm{L}^{2}(\Omega)$, let $\left(\psi_{1}, \psi_{2}\right)$ be the solution of (3.14) with $\left(\tau^{0}, \theta_{2} \tau^{0}\right)$ as final data. Suppose $z$ is the solution of the system:

$$
\begin{cases}\theta_{1} z_{1}{ }^{\prime}-\operatorname{div}\left(A^{0} \nabla z_{1}\right)+c_{h}\left(\theta_{2} z_{1}-z_{2}\right)=\chi_{\omega} \theta_{1} \widehat{\varphi}_{1} & \text { in } \Omega \times(0, T), \\ z_{2}{ }^{\prime}-c_{h}\left(\theta_{2} z_{1}-z_{2}\right)=\chi_{\omega} \widehat{\varphi}_{2} & \text { in } \Omega \times(0, T), \\ z_{1}=0 & \text { on } \partial \Omega \times(0, T), \\ z_{1}(x, 0)=0, \quad z_{2}(x, 0)=0 & \text { in } \Omega .\end{cases}
$$

Choosing $\left(\psi_{1}, 0\right)$ as test function in (4.9) and integrating by parts, we get

$$
\begin{gathered}
-\int_{0}^{T} \theta_{1}\left\langle z_{1}, \psi_{1}^{\prime}\right\rangle_{\mathrm{L}^{2}(\Omega), \mathrm{L}^{2}(\Omega)} \mathrm{d} t+\int_{\Omega} \theta_{1} z_{1}(T) \psi_{1}(T) \mathrm{d} x+\int_{0}^{T} \int_{\Omega} A^{0} \nabla z_{1} \nabla \psi_{1} \mathrm{~d} x \mathrm{~d} t \\
+\int_{0}^{T} \int_{\Omega} c_{h}\left(\theta_{2} z_{1}-z_{2}\right) \psi_{1} \mathrm{~d} x \mathrm{~d} t=\int_{0}^{T} \int_{\omega} \theta_{1} \widehat{\varphi}_{1} \psi_{1} \mathrm{~d} x \mathrm{~d} t .
\end{gathered}
$$

Now, choosing $\left(z_{1}, 0\right)$ as test function in (3.14) (written for $\psi$ ),

$-\int_{0}^{T} \theta_{1}\left\langle\psi_{1}^{\prime}, z_{1}\right\rangle_{\mathrm{L}^{2}(\Omega), \mathrm{L}^{2}(\Omega)} \mathrm{d} t+\int_{0}^{T} \int_{\Omega} A^{0} \nabla \psi_{1} \nabla z_{1} \mathrm{~d} x \mathrm{~d} t+\int_{0}^{T} \int_{\Omega} c_{h}\left(\theta_{2} \psi_{1}-\psi_{2}\right) z_{1} \mathrm{~d} x \mathrm{~d} t=0$.

Subtracting (4.11) from (4.10), from the symmetry of $A^{0}$ and the fact that $\psi_{1}(T)=\tau^{0}$, we have

$$
\int_{\Omega} \theta_{1} z_{1}(T) \tau^{0} \mathrm{~d} x+\int_{0}^{T} \int_{\Omega} c_{h}\left(z_{1} \psi_{2}-z_{2} \psi_{1}\right) \mathrm{d} x \mathrm{~d} t=\int_{0}^{T} \int_{\omega} \theta_{1} \widehat{\varphi}_{1} \psi_{1} \mathrm{~d} x \mathrm{~d} t .
$$

Let us now take $\left(0, \psi_{2}\right)$ as test function in (4.9) and integrate by parts so that

$-\int_{0}^{T}\left\langle z_{2}, \psi_{2}^{\prime}\right\rangle_{\mathrm{L}^{2}(\Omega), \mathrm{L}^{2}(\Omega)} \mathrm{d} t+\int_{\Omega} z_{2}(T) \psi_{2}(T) \mathrm{d} x-\int_{0}^{T} \int_{\Omega} c_{h}\left(\theta_{2} z_{1}-z_{2}\right) \psi_{2} \mathrm{~d} x \mathrm{~d} t=\int_{0}^{T} \int_{\omega} \widehat{\varphi}_{2} \psi_{2} \mathrm{~d} x \mathrm{~d} t$.

On the other hand, choosing $\left(0, z_{2}\right)$ as test function in (3.14) (written for $\psi$ ) gives

$$
-\int_{0}^{T}\left\langle\psi_{2}^{\prime}, z_{2}\right\rangle_{\mathrm{L}^{2}(\Omega), \mathrm{L}^{2}(\Omega)} \mathrm{d} t-\int_{0}^{T} \int_{\Omega} c_{h}\left(\theta_{2} \psi_{1}-\psi_{2}\right) z_{2} \mathrm{~d} x \mathrm{~d} t=0 .
$$

Subtracting (4.14) from (4.13) and using the fact that $\psi_{2}(T)=\theta_{2} \tau^{0}$, we have

$$
\int_{\Omega} z_{2}(T) \theta_{2} \tau^{0} \mathrm{~d} x+\int_{0}^{T} \int_{\Omega} c_{h}\left(\theta_{2} \psi_{1} z_{2}-\theta_{2} \psi_{2} z_{1}\right) \mathrm{d} x \mathrm{~d} t=\int_{0}^{T} \int_{\omega} \widehat{\varphi}_{2} \psi_{2} \mathrm{~d} x \mathrm{~d} t
$$

It follows that

$$
\int_{\Omega} z_{2}(T) \tau^{0} \mathrm{~d} x+\int_{0}^{T} \int_{\Omega} c_{h}\left(\psi_{1} z_{2}-\psi_{2} z_{1}\right) \mathrm{d} x \mathrm{~d} t=\theta_{2}^{-1} \int_{0}^{T} \int_{\omega} \widehat{\varphi}_{2} \psi_{2} \mathrm{~d} x \mathrm{~d} t .
$$

Adding (4.12) and (4.16) gives

$$
\int_{\Omega} \theta_{1} z_{1}(T) \tau^{0} \mathrm{~d} x+\int_{\Omega} z_{2}(T) \tau^{0} \mathrm{~d} x=\theta_{1} \int_{0}^{T} \int_{\omega} \widehat{\varphi}_{1} \psi_{1} \mathrm{~d} x \mathrm{~d} t+\theta_{2}^{-1} \int_{0}^{T} \int_{\omega} \widehat{\varphi}_{2} \psi_{2} \mathrm{~d} x \mathrm{~d} t
$$

while substituting (4.17) in (4.8) yields

$$
\begin{aligned}
& \left|\int_{\Omega} \theta_{1} z_{1}(T) \tau^{0} \mathrm{~d} x+\int_{\Omega} z_{2}(T) \tau^{0} \mathrm{~d} x-\int_{\Omega}\left(w-v_{1}(T)\right) \theta_{1} \tau^{0} \mathrm{~d} x-\int_{\Omega}\left(w-\theta_{2}^{-1} v_{2}(T)\right) \theta_{2} \tau^{0} \mathrm{~d} x\right| \\
& \leq\left(\delta_{1} \sqrt{\theta_{1}}+\delta_{2} \sqrt{\theta_{2}}\right)\left\|\tau^{0}\right\|_{\mathrm{L}^{2}(\Omega)} .
\end{aligned}
$$

This proves (3.16), since by the definition of $u$ and $v$ one has $u_{i}(T)=z_{i}(T)+v_{i}(T)$, for $i=1,2$. 


\section{Proof of THE CONTROL FOR FIXED $\varepsilon$}

In this section, we show the approximate controllability of system (2.2) for fixed $\varepsilon$, stated in Theorem 3.2, using the variational approach, as in the preceding section.

Proposition 5.1. Under assumptions (2.4)-(2.8), (3.17) and (3.24), let $J_{\varepsilon}$ be the functional defined by (3.1). Then, for every fixed $\varepsilon$, we have

$$
\liminf _{\left\|\varphi_{\varepsilon}^{0}\right\|_{L^{2}(\Omega)} \rightarrow \infty} \frac{J_{\varepsilon}\left(\varphi_{\varepsilon}^{0}\right)}{\left\|\varphi_{\varepsilon}^{0}\right\|_{\mathrm{L}^{2}(\Omega)}} \geq \min \left\{\delta_{1}, \delta_{2}\right\}
$$

Proof. The proof follows the same outline as that of the proof to show (4.1). The only point to be checked is the fact that if the solution $\left(\psi_{1}, \psi_{2}\right)$ of the adjoint problem $(3.2)$ verifies $\psi_{1}=0$, in $\omega_{1} \times(0, T)$ and $\psi_{2}=$ 0 , in $\omega_{2} \times(0, T)$, then $\psi_{i}$ is zero in $\Omega_{i} \times(0, T), i=1,2$.

To prove that, we observe that by assumptions (2.3) and (2.8), we can apply the unique-continuation property of [12] to the problem satisfied by $\psi_{1}$ in $\omega_{1} \times(0, T)$, which has a unique solution. Then, arguing as in the proof of Corollary 1.2 of [12] we get $\psi_{1}=0$ in $\Omega_{1} \times(0, T)$. Consequently, we also have $\psi_{2}$ is zero in $\Omega_{2} \times(0, T)$.

Remark 5.2. Observe that by standard arguments, $J_{\varepsilon}$ is continuous and strictly convex. Together with Proposition 5.1, this implies that $J_{\varepsilon}$ has a unique minimum point, $\widehat{\varphi}_{\varepsilon}^{0} \in \mathrm{L}^{2}(\Omega)$.

The following result can be deduced by similar arguments as those used to show (4.4) and (4.8).

Proposition 5.3. Let $\psi_{\varepsilon}^{0} \in \mathrm{L}^{2}(\Omega)$ and $i=1,2$ and suppose $(2.4)-(2.8)$. If $\widehat{\varphi}_{\varepsilon}^{0}$ is the minimum point of the functional $J_{\varepsilon}$, then

$$
\left|\sum_{i=1}^{2}\left(\int_{0}^{T} \int_{\omega_{i}} \widehat{\varphi}_{i \varepsilon} \psi_{i \varepsilon} \mathrm{d} x \mathrm{~d} t-\int_{\Omega_{i \varepsilon}}\left(w_{\varepsilon}-v_{i \varepsilon}(T)\right) \psi_{\varepsilon}^{0} \mathrm{~d} x\right)\right| \leq \delta_{1}\left\|\psi_{\varepsilon}^{0}\right\|_{\mathrm{L}^{2}\left(\Omega_{1 \varepsilon}\right)}+\delta_{2}\left\|\psi_{\varepsilon}^{0}\right\|_{\mathrm{L}^{2}\left(\Omega_{2 \varepsilon}\right)},
$$

where $\widehat{\varphi}_{\varepsilon}=\left(\widehat{\varphi}_{1 \varepsilon}, \widehat{\varphi}_{2 \varepsilon}\right)$ is the solution of $(3.2)$ with the corresponding final data $\widehat{\varphi}_{\varepsilon}^{0}$.

Proof of Theorem 3.2. The proof is analogous to those of Theorems 3.3 and 3.4. In the same way, to prove (3.6), we decompose the solution $u_{\varepsilon}$ as $u_{\varepsilon}=z_{\varepsilon}+v_{\varepsilon}$ where $v_{\varepsilon}$ is defined by $(3.3)$ and $z_{\varepsilon}=\left(z_{1 \varepsilon}, z_{2 \varepsilon}\right)$ is the solution of problem (3.5) corresponding to the initial data $U_{\varepsilon}^{0}=0$.

Let $\psi_{\varepsilon}^{0} \in \mathrm{L}^{2}(\Omega)$ and suppose $\psi_{\varepsilon}=\left(\psi_{1 \varepsilon}, \psi_{2 \varepsilon}\right)$ is the solution of the transposed problem (3.2) with final data $\psi_{\varepsilon}^{0}$. We choose $\psi_{\varepsilon}$ as test functions in the problem solved by $z_{\varepsilon}$ and and $z_{\varepsilon}$ as test function in (3.2) (written for $\left.\psi_{\varepsilon}\right)$. Using similar arguments to show (4.17), since $\psi_{\varepsilon}(x, T)=\psi_{\varepsilon}^{0}$ and $A^{\varepsilon}$ is symmetric, we deduce that

$$
\int_{0}^{T} \int_{\omega_{1 \varepsilon}} \widehat{\varphi}_{1 \varepsilon} \psi_{1 \varepsilon} \mathrm{d} x \mathrm{~d} t+\int_{0}^{T} \int_{\omega_{2 \varepsilon}} \widehat{\varphi}_{2 \varepsilon} \psi_{2 \varepsilon} \mathrm{d} x \mathrm{~d} t=\int_{\Omega_{1 \varepsilon}} z_{1 \varepsilon}(T) \psi_{\varepsilon}^{0} \mathrm{~d} x+\int_{\Omega_{2 \varepsilon}} z_{2 \varepsilon}(T) \psi_{\varepsilon}^{0} \mathrm{~d} x .
$$

Together with Proposition 5.3 and using the decomposition $u_{\varepsilon}=z_{\varepsilon}+v_{\varepsilon}$, we get

$$
\left|\int_{\Omega_{1 \varepsilon}}\left(u_{1 \varepsilon}(T)-w_{\varepsilon}\right) \psi_{\varepsilon}^{0} \mathrm{~d} x+\int_{\Omega_{2 \varepsilon}}\left(u_{2 \varepsilon}(T)-w_{\varepsilon}\right) \psi_{\varepsilon}^{0} \mathrm{~d} x\right| \leq \delta_{1}\left\|\psi_{\varepsilon}^{0}\right\|_{\mathrm{L}^{2}\left(\Omega_{1 \varepsilon}\right)}+\delta_{2}\left\|\psi_{\varepsilon}^{0}\right\|_{\mathrm{L}^{2}\left(\Omega_{2 \varepsilon}\right)} .
$$

Taking $\psi_{\varepsilon}^{0}=0$ in $\Omega_{2 \varepsilon}$ gives

$$
\left|\int_{\Omega_{1 \varepsilon}}\left(u_{1 \varepsilon}(T)-w_{\varepsilon}\right) \psi_{\varepsilon}^{0} \mathrm{~d} x\right| \leq \delta_{1}\left\|\psi_{\varepsilon}^{0}\right\|_{\mathrm{L}^{2}\left(\Omega_{1 \varepsilon}\right)}, \forall \psi_{\varepsilon}^{0} \in \mathrm{L}^{2}\left(\Omega_{1 \varepsilon}\right)
$$

so that by definition, we obtain (3.6) for $i=1$. Similarly, choosing $\psi_{\varepsilon}^{0}=0$ in $\Omega_{1 \varepsilon}$ in (5.4), we obtain (3.6) for $i=2$. This completes the result. 


\section{Proof of the main Convergence Result}

In order to prove the main convergence result given by Theorem 3.5 we need to prove first the next proposition which, as in [13] for the case of rapidly oscillating coefficients in a fixed domain and in [7] for perforated domains, provides a uniform estimate in $\varepsilon$ for the unique minimum point $\widehat{\varphi}_{\varepsilon}^{0}$ of the functional $J_{\varepsilon}$.

Proposition 6.1. Under the assumptions of Theorem 3.5, the functional $J_{\varepsilon}$ defined by (3.1) satisfies,

$$
\liminf _{\left\|\varphi_{\varepsilon}^{0}\right\|_{L_{L^{2}(\Omega)} \rightarrow \infty} \infty} \frac{J_{\varepsilon}\left(\varphi_{\varepsilon}^{0}\right)}{\left\|\varphi_{\varepsilon}^{0}\right\|_{L^{2}(\Omega)}} \geq \min \left\{\delta_{1}, \delta_{2}\right\} .
$$

Proof. Let $\left\{\varepsilon_{j}\right\}_{j \in \mathbb{N}}$ be a subsequence of $\{\varepsilon\}_{\varepsilon>0}$ and $\varphi_{\varepsilon_{j}}^{0} \in \mathrm{L}^{2}(\Omega)$ a corresponding sequence, denoted for simplicity $\varphi_{\varepsilon}^{0} \in \mathrm{L}^{2}(\Omega)$ in the sequel, such that $\left\|\varphi_{\varepsilon}^{0}\right\|_{\mathrm{L}^{2}(\Omega)} \rightarrow \infty$ as $\varepsilon \rightarrow 0$. Set

$$
\bar{\varphi}_{\varepsilon}^{0}=\left(\left.\bar{\varphi}_{\varepsilon}^{0}\right|_{\Omega_{1 \varepsilon}},\left.\bar{\varphi}_{\varepsilon}^{0}\right|_{\Omega_{2 \varepsilon}}\right)=\frac{\varphi_{\varepsilon}^{0}}{\left\|\varphi_{\varepsilon}^{0}\right\|_{L^{2}(\Omega)}}, \quad \bar{\varphi}_{\varepsilon}=\left(\bar{\varphi}_{1 \varepsilon}, \bar{\varphi}_{2 \varepsilon}\right)=\frac{\varphi_{\varepsilon}}{\left\|\varphi_{\varepsilon}^{0}\right\|_{L^{2}(\Omega)}},
$$

where $\varphi_{\varepsilon}=\left(\varphi_{1 \varepsilon}, \varphi_{2 \varepsilon}\right)$ is the solution of the adjoint problem (3.2) with final data $\varphi_{\varepsilon}^{0}$.

Observe that there exists a subsequence (still denoted by $\varepsilon$ ) and $\sigma^{0}, \tau^{0} \in \mathrm{L}^{2}(\Omega)$ such that

$$
\left(\chi_{\Omega_{1 \varepsilon}} \bar{\varphi}_{\varepsilon}^{0}, \chi_{\Omega_{2 \varepsilon}} \bar{\varphi}_{\varepsilon}^{0}\right) \rightarrow\left(\theta_{1} \sigma^{0}, \theta_{2} \tau^{0}\right) \quad \text { weakly in }\left[\mathrm{L}^{2}(\Omega)\right]^{2} .
$$

Take note also that $\bar{\varphi}_{\varepsilon}$ satisfies (3.2) written for the final data $\bar{\varphi}_{\varepsilon}^{0}$, which applying Theorem 2.2 gives

$$
\left(\widetilde{\overline{\varphi_{1 \varepsilon}}}, \widetilde{\bar{\varphi}_{2 \varepsilon}}\right) \rightarrow\left(\theta_{1} \bar{\varphi}_{1}, \bar{\varphi}_{2}\right) \quad \text { weakly* in } \mathrm{L}^{\infty}\left(0, T ; \mathrm{L}^{2}(\Omega)\right) \times \mathrm{L}^{\infty}\left(0, T ; \mathrm{L}^{2}(\Omega)\right) .
$$

Case $-\mathbf{1}<\boldsymbol{\gamma}<\mathbf{1}$ : The limit $\bar{\varphi}_{2}$ is given by $\bar{\varphi}_{2}=\theta_{2} \bar{\varphi}_{1}$, where $\bar{\varphi}_{1}$ is the solution of

$$
\begin{cases}-\bar{\varphi}_{1}^{\prime}-\operatorname{div}\left(A^{0} \nabla \bar{\varphi}_{1}\right)=0 & \text { in } \Omega \times(0, T), \\ \bar{\varphi}_{1}=0 & \text { on } \partial \Omega \times(0, T), \\ \bar{\varphi}_{1}(x, T)=\theta_{1} \sigma^{0}+\theta_{2} \tau^{0} & \text { in } \Omega .\end{cases}
$$

Case $\gamma=1$ : The limit $\left(\bar{\varphi}_{1}, \bar{\varphi}_{2}\right)$ is the solution to the following equation:

$$
\begin{cases}-\theta_{1} \bar{\varphi}_{1}^{\prime}-\operatorname{div}\left(A^{0} \nabla \bar{\varphi}_{1}\right)+c_{h}\left(\theta_{2} \bar{\varphi}_{1}-\bar{\varphi}_{2}\right)=0 & \text { in } \Omega \times(0, T), \\ -\bar{\varphi}_{2}^{\prime}-c_{h}\left(\theta_{2} \bar{\varphi}_{1}-\bar{\varphi}_{2}\right)=0 & \text { in } \Omega \times(0, T), \\ \bar{\varphi}_{1}=0 & \text { on } \partial \Omega \times(0, T), \\ \bar{\varphi}_{1}(x, T)=\sigma^{0}, \quad \bar{\varphi}_{2}(x, T)=\theta_{2} \tau^{0} & \text { in } \Omega .\end{cases}
$$

Now, by the definition of $J_{\varepsilon}$ given in (3.1),

$$
\begin{aligned}
\frac{J_{\varepsilon}\left(\varphi_{\varepsilon}^{0}\right)}{\left\|\varphi_{\varepsilon}^{0}\right\|_{\mathrm{L}^{2}(\Omega)}}= & \frac{1}{2}\left\|\varphi_{\varepsilon}^{0}\right\|_{\mathrm{L}^{2}(\Omega)}\left(\int_{0}^{T} \int_{\omega_{1 \varepsilon}}\left|\bar{\varphi}_{1 \varepsilon}\right|^{2} \mathrm{~d} x \mathrm{~d} t+\int_{0}^{T} \int_{\omega_{2 \varepsilon}}\left|\bar{\varphi}_{2 \varepsilon}\right|^{2} \mathrm{~d} x \mathrm{~d} t\right)+\delta_{1}\left\|\bar{\varphi}_{\varepsilon}^{0}\right\|_{\mathrm{L}^{2}\left(\Omega_{1 \varepsilon}\right)} \\
& +\delta_{2}\left\|\bar{\varphi}_{\varepsilon}^{0}\right\|_{\mathrm{L}^{2}\left(\Omega_{2 \varepsilon}\right)}-\int_{\Omega_{1 \varepsilon}}\left(w_{\varepsilon}-v_{1 \varepsilon}(T)\right) \bar{\varphi}_{\varepsilon}^{0} \mathrm{~d} x-\int_{\Omega_{2 \varepsilon}}\left(w_{\varepsilon}-v_{2 \varepsilon}(T)\right) \bar{\varphi}_{\varepsilon}^{0} \mathrm{~d} x .
\end{aligned}
$$


Case 1. At least one of the $\liminf _{\varepsilon \rightarrow 0} \int_{0}^{T} \int_{\omega_{i \varepsilon}}\left|\bar{\varphi}_{i \varepsilon}\right|^{2} \mathrm{~d} x \mathrm{~d} t, i=1,2$ is strictly positive.

Using our assumption that $\left\|\varphi_{\varepsilon}^{0}\right\|_{\mathrm{L}^{2}(\Omega)} \rightarrow \infty$, we have

$$
\left\|\varphi_{\varepsilon}^{0}\right\|_{\mathrm{L}^{2}(\Omega)}\left(\int_{0}^{T} \int_{\omega_{1 \varepsilon}}\left|\bar{\varphi}_{1 \varepsilon}\right|^{2} \mathrm{~d} x \mathrm{~d} t+\int_{0}^{T} \int_{\omega_{2 \varepsilon}}\left|\bar{\varphi}_{2 \varepsilon}\right|^{2} \mathrm{~d} x \mathrm{~d} t\right) \rightarrow \infty .
$$

On the other hand, by (3.29)-(3.31) and (6.2), $\left\|w_{\varepsilon}-v_{i \varepsilon}(T)\right\|_{\mathrm{L}^{2}\left(\Omega_{i \varepsilon}\right)}<\infty$ and $\left\|\bar{\varphi}_{\varepsilon}^{0}\right\|_{\mathrm{L}^{2}\left(\Omega_{i \varepsilon}\right)}<\infty$ so that, by Hölder inequality, the last two terms of (6.7) are bounded. Lastly, since $\left\|\bar{\varphi}_{\varepsilon}^{0}\right\|_{\mathrm{L}^{2}\left(\Omega_{i \varepsilon}\right)} \leq 1, i=1,2$, we have $\delta_{1}\left\|\bar{\varphi}_{\varepsilon}^{0}\right\|_{L^{2}\left(\Omega_{1 \varepsilon}\right)}+\delta_{2}\left\|\bar{\varphi}_{\varepsilon}^{0}\right\|_{L^{2}\left(\Omega_{2 \varepsilon}\right)} \leq \delta_{1}+\delta_{2}$. Therefore, the limit in (6.1) is equal to $+\infty$, which gives the estimate.

Case 2. $\liminf _{\varepsilon \rightarrow 0} \int_{0}^{T} \int_{\omega_{1 \varepsilon}}\left|\bar{\varphi}_{1 \varepsilon}\right|^{2} \mathrm{~d} x \mathrm{~d} t=\liminf _{\varepsilon \rightarrow 0} \int_{0}^{T} \int_{\omega_{2 \varepsilon}}\left|\bar{\varphi}_{2 \varepsilon}\right|^{2} \mathrm{~d} x \mathrm{~d} t=0$. From (6.4) and our assumption, we deduce that $\bar{\varphi}_{1}=\bar{\varphi}_{2}=0$ in $\omega \times(0, T)$.

If $-1<\gamma<1$, from (6.5) and the unique-continuation property from [12], we have $\bar{\varphi}_{1}=\bar{\varphi}_{2}=0$ in $\Omega \times(0, T)$. Since $\left.\bar{\varphi}_{1} \in \mathrm{C}^{0}\left([0, T] ; \mathrm{L}^{2}(\Omega)\right)\right)$, it follows that $\bar{\varphi}_{1}(T)=\theta_{1} \sigma^{0}+\theta_{2} \tau^{0}=0$ in $\mathrm{L}^{2}(\Omega)$. Hence, from (6.3), the function $\bar{\varphi}_{\varepsilon}^{0}$ weakly converges to zero in $\mathrm{L}^{2}(\Omega)$. This, together with (3.29), yields

$$
\lim _{\varepsilon \rightarrow 0}\left(\int_{\Omega_{1 \varepsilon}}\left(w_{\varepsilon}-v_{1 \varepsilon}(T)\right) \bar{\varphi}_{\varepsilon}^{0} \mathrm{~d} x+\int_{\Omega_{2 \varepsilon}}\left(w_{\varepsilon}-v_{2 \varepsilon}(T)\right) \bar{\varphi}_{\varepsilon}^{0} \mathrm{~d} x\right)=\lim _{\varepsilon \rightarrow 0} \int_{\Omega}\left(w_{\varepsilon}-v_{\varepsilon}(T)\right) \bar{\varphi}_{\varepsilon}^{0} \mathrm{~d} x=0 .
$$

Now, let $\gamma=1$. Since the couple $\left(\bar{\varphi}_{1}, \bar{\varphi}_{2}\right)$ satisfies (6.6), by Theorem 7.1 of the Appendix we derive that $\bar{\varphi}_{1}=\bar{\varphi}_{2}=0$ in $\Omega \times(0, T)$. This implies, since $\bar{\varphi}_{1}, \bar{\varphi}_{2} \in \mathrm{C}^{0}\left([0, T] ; \mathrm{L}^{2}(\Omega)\right)$,

$$
\left\{\begin{array}{l}
\bar{\varphi}_{1}(T)=\sigma^{0}=0 \quad \text { in } \mathrm{L}^{2}(\Omega) \\
\bar{\varphi}_{2}(T)=\theta_{2} \tau^{0}=0 \quad \text { in } \mathrm{L}^{2}(\Omega) .
\end{array}\right.
$$

Thus, for $\gamma=1$, arguing as done above for the previous case, we have

$$
\left(\chi_{\Omega_{1 \varepsilon}} \bar{\varphi}_{\varepsilon}^{0}, \chi_{\Omega_{2 \varepsilon}} \bar{\varphi}_{\varepsilon}^{0}\right) \rightarrow(0,0) \quad \text { weakly in }\left[\mathrm{L}^{2}(\Omega)\right]^{2} .
$$

Using (3.31), this yields

$$
\begin{aligned}
& \lim _{\varepsilon \rightarrow 0}\left(\int_{\Omega_{1 \varepsilon}}\left(w_{\varepsilon}-v_{1 \varepsilon}(T)\right) \bar{\varphi}_{\varepsilon}^{0} \mathrm{~d} x+\int_{\Omega_{2 \varepsilon}}\left(w_{\varepsilon}-v_{2 \varepsilon}(T)\right) \bar{\varphi}_{\varepsilon}^{0} \mathrm{~d} x\right) \\
& =\lim _{\varepsilon \rightarrow 0}\left(\int_{\Omega_{1 \varepsilon}}\left[\left(w_{\varepsilon}-v_{1 \varepsilon}(T)\right)-\left(w-v_{1}(T)\right)\right] \bar{\varphi}_{\varepsilon}^{0} \mathrm{~d} x+\int_{\Omega}\left(w-v_{1}(T)\right) \chi_{\Omega_{1 \varepsilon}} \bar{\varphi}_{\varepsilon}^{0} \mathrm{~d} x\right) \\
& \quad+\lim _{\varepsilon \rightarrow 0}\left(\int_{\Omega_{2 \varepsilon}}\left[\left(w_{\varepsilon}-v_{2 \varepsilon}(T)\right)-\left(w-\theta_{2}^{-1} v_{2}(T)\right)\right] \bar{\varphi}_{\varepsilon}^{0} \mathrm{~d} x+\int_{\Omega}\left(w-\theta_{2}^{-1} v_{2}(T)\right) \chi_{\Omega_{2 \varepsilon}} \bar{\varphi}_{\varepsilon}^{0} \mathrm{~d} x\right)=0 .
\end{aligned}
$$

Lastly,

$$
\lim _{\varepsilon \rightarrow 0}\left(\delta_{1}\left\|\bar{\varphi}_{\varepsilon}^{0}\right\|_{\mathrm{L}^{2}\left(\Omega_{1 \varepsilon}\right)}+\delta_{2}\left\|\bar{\varphi}_{\varepsilon}^{0}\right\|_{\mathrm{L}^{2}\left(\Omega_{2 \varepsilon}\right)}\right)=\lim _{\varepsilon \rightarrow 0} \frac{\delta_{1}\left\|\varphi_{\varepsilon}^{0}\right\|_{\mathrm{L}^{2}\left(\Omega_{1 \varepsilon}\right)}+\delta_{2}\left\|\varphi_{\varepsilon}^{0}\right\|_{\mathrm{L}^{2}\left(\Omega_{2 \varepsilon}\right)}}{\left(\left\|\varphi_{\varepsilon}^{0}\right\|_{\mathrm{L}^{2}\left(\Omega_{1 \varepsilon}\right)}^{2}+\left\|\varphi_{\varepsilon}^{0}\right\|_{\mathrm{L}^{2}\left(\Omega_{2 \varepsilon}\right)}^{2}\right.} \geq \min \left\{\delta_{1}, \delta_{2}\right\} .
$$

Using (6.8), (6.11), (6.12) and the assumption done for this case, we have the claimed estimate (6.1).

Corollary 6.2. Under the assumptions of Theorem 3.5, let $\widehat{\varphi}_{\varepsilon}^{0}$ be defined in Theorem 3.2. Then, there exists a constant $C$ independent of $\varepsilon$ such that

$$
\left\|\widehat{\varphi}_{\varepsilon}^{0}\right\|_{L^{2}(\Omega)} \leq C
$$


Proof. We prove the result by contradiction. Suppose there exists a subsequence (still denoted $\varepsilon$ ) such that $\left\|\widehat{\varphi}_{\varepsilon}^{0}\right\|_{L^{2}(\Omega)} \rightarrow \infty$, as $\varepsilon \rightarrow 0$. Since $\widehat{\varphi}_{\varepsilon}^{0}$ is the minimum point of the functional $J_{\varepsilon}$, one has $J_{\varepsilon}\left(\widehat{\varphi}_{\varepsilon}^{0}\right) \leq J_{\varepsilon}(0)=0$, for any $\varepsilon$. Hence, by Proposition 6.1, we obtain a contradiction, since

$$
0<\min \left\{\delta_{1}, \delta_{2}\right\} \leq \liminf _{\varepsilon \rightarrow 0} \frac{J_{\varepsilon}\left(\widehat{\varphi}_{\varepsilon}^{0}\right)}{\left\|\widehat{\varphi}_{\varepsilon}^{0}\right\|_{\mathrm{L}^{2}(\Omega)}} \leq 0 .
$$

Remark 6.3. From Corollary 6.2, there exists $\left(\xi^{0}, \nu^{0}\right) \in\left[\mathrm{L}^{2}(\Omega)\right]^{2}$ such that (up to a subsequence)

$$
\left\{\left(\chi_{\Omega_{1 \varepsilon}} \widehat{\varphi}_{\varepsilon}^{0}, \chi_{\Omega_{2 \varepsilon}} \widehat{\varphi}_{\varepsilon}^{0}\right)\right\}_{\varepsilon} \rightarrow\left(\theta_{1} \xi^{0}, \theta_{2} \nu^{0}\right) \quad \text { weakly in }\left[\mathrm{L}^{2}(\Omega)\right]^{2} .
$$

The next theorem identifies the weak limit $\left(\xi^{0}, \nu^{0}\right)$ in $(6.14)$, precise some behavior of the controls and lead us to the proof of our main result, Theorem 3.5. It is proved at the end of this section after several preliminary results, by adapting to our case some techniques used by Zuazua [13,14] and Donato and Nabil [7].

Theorem 6.4. Let $U^{0}$ and $w$ be given in $\mathrm{L}^{2}(\Omega)$. Under the assumptions of Theorem 3.5 , let $\widehat{\varphi}_{\varepsilon}^{0}$ be the minimum point of $J_{\varepsilon}$, and $\left(\widehat{\Phi}^{0}, \widehat{\Psi}^{0}\right)$ the unique minimum point of the functional $J_{0}$ given by (3.8) and (3.13) according on $\gamma$. Then, as $\varepsilon \rightarrow 0$,

$$
\left(\chi_{\Omega_{1 \varepsilon}} \widehat{\varphi}_{\varepsilon}^{0}, \chi_{\Omega_{2 \varepsilon}} \widehat{\varphi}_{\varepsilon}^{0}\right) \rightarrow\left(\theta_{1} \widehat{\Phi}^{0}, \theta_{2} \widehat{\Psi}^{0}\right) \quad \text { weakly in }\left[\mathrm{L}^{2}(\Omega)\right]^{2}
$$

Corollary 6.5. Under the hypothesis of Theorem 3.5, let $\widehat{\varphi}_{\varepsilon}$ be the control $\widehat{\varphi}_{\varepsilon}$ given by Theorem 3.2.

Case $-\mathbf{1}<\gamma<\mathbf{1}$ : Let $\widehat{\varphi}$ be the solution of (3.9) with final data $\theta_{1} \widehat{\Phi}^{0}+\theta_{2} \widehat{\Psi}^{0}$, where the pair $\left(\widehat{\Phi}^{0}, \widehat{\Psi}^{0}\right)$ is the unique minimum point of the functional $J_{0}$ defined by (3.8). Then, as $\varepsilon \rightarrow 0$,

$$
\left\{\begin{array}{lll}
\text { (i) } & \widehat{\varphi}_{\varepsilon} \rightarrow \widehat{\varphi} & \text { strongly in } \mathrm{L}^{2}\left(0, T ; \mathrm{L}^{2}(\Omega)\right), \\
\text { (ii) } & P_{1}^{\varepsilon} \widehat{\varphi}_{1 \varepsilon} \rightarrow \widehat{\varphi} & \text { weakly in } \mathrm{L}^{2}\left(0, T ; \mathrm{H}_{0}^{1}(\Omega)\right),
\end{array}\right.
$$

Case $\gamma=1$ : Let $\left(\widehat{\varphi}_{1}, \widehat{\varphi}_{2}\right)$ be the solution of (3.14) with final data $\left(\widehat{\Phi}^{0}, \theta_{2} \widehat{\Psi}^{0}\right)$, where $\left(\widehat{\Phi}^{0}, \widehat{\Psi}^{0}\right)$ is the unique minimum point of the functional $J_{0}$ defined by (3.13). Then, as $\varepsilon \rightarrow 0$,

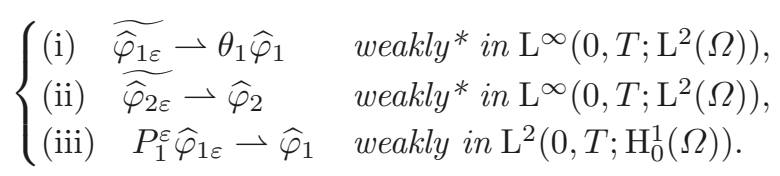

Proof. Convergences (6.16) and (6.17) directly follow from (6.15) by applying Theorem 2.2 to the control $\widehat{\varphi}_{\varepsilon}$ with the aid of Proposition 2.4 to show (6.16)(i).

In the two propositions below, we state a convergence result which is an essential tool for proving Theorem 6.4. Although the results are similar in the two cases, we state them separately, since the proofs are different.

Proposition 6.6. Let $-1<\gamma<1$. Suppose (2.4)-(2.7) and (3.17) hold. Then the functional $J_{\varepsilon}$ defined by (3.1) satisfies

$$
\lim _{\varepsilon \rightarrow 0} J_{\varepsilon}\left(\chi_{\Omega_{1 \varepsilon}} \Phi^{0}+\chi_{\Omega_{2 \varepsilon}} \Psi^{0}\right)=J_{0}\left(\Phi^{0}, \Psi^{0}\right)
$$

for any $\Phi_{0}$ and $\Psi^{0}$ in $\mathrm{L}^{2}(\Omega)$, where $J_{0}$ is the functional given by (3.8). 
Proof. By definition (see Rem. 3.1),

$$
\begin{aligned}
J_{\varepsilon}\left(\chi_{\Omega_{1 \varepsilon}} \Phi^{0}+\chi_{\Omega_{2 \varepsilon}} \Psi^{0}\right)= & \frac{1}{2} \int_{0}^{T} \int_{\omega}\left|\psi_{\varepsilon}\right|^{2} \mathrm{~d} x \mathrm{~d} t+\delta_{1}\left\|\Phi^{0}\right\|_{\mathrm{L}^{2}\left(\Omega_{1 \varepsilon}\right)}+\delta_{2}\left\|\Psi^{0}\right\|_{\mathrm{L}^{2}\left(\Omega_{2 \varepsilon}\right)} \\
& -\int_{\Omega_{1 \varepsilon}}\left(w_{\varepsilon}-v_{1 \varepsilon}(T)\right) \Phi^{0} \mathrm{~d} x-\int_{\Omega_{2 \varepsilon}}\left(w_{\varepsilon}-v_{2 \varepsilon}(T)\right) \Psi^{0} \mathrm{~d} x
\end{aligned}
$$

for any $\Phi_{0}$ and $\Psi^{0}$ in $\mathrm{L}^{2}(\Omega)$, where $\psi_{\varepsilon}(x, t)$ is the solution of problem (3.2) with final data $\chi_{\Omega_{1 \varepsilon}} \Phi^{0}+\chi_{\Omega_{2 \varepsilon}} \Psi^{0}$, and $v_{\varepsilon}=\left(v_{1 \varepsilon}, v_{2 \varepsilon}\right)$ is the solution of the auxiliary problem (3.3).

Since $\left(\chi_{\Omega_{1 \varepsilon}} \Phi^{0}, \chi_{\Omega_{2 \varepsilon}} \Psi^{0}\right) \rightarrow\left(\theta_{1} \Phi^{0}, \theta_{2} \Psi^{0}\right)$ weakly in $\left[\mathrm{L}^{2}(\Omega)\right]^{2}$, Theorem 2.2 and Proposition 2.4 applied to $\psi_{\varepsilon}$, imply that $\psi_{\varepsilon} \rightarrow \psi_{1}$ strongly in $\mathrm{L}^{2}\left(0, T ; \mathrm{L}^{2}(\Omega)\right)$, where $\psi_{1}$ is the solution of (3.9). This, together with (2.1) and (3.29), allows us to pass to the limit in (6.19) to obtain the desired result.

The next proposition concern the case $\gamma=1$, where the homogenized problem contains a coupled system of two equations.

Proposition 6.7. Let $\gamma=1$ and assume that $\Gamma$ is of class $\mathcal{C}^{2}$. For $\Phi^{0}, \Psi^{0} \in \mathrm{L}^{2}(\Omega)$, suppose that (2.4)-(2.7) and (3.24) hold. Then the functional $J_{\varepsilon}$ satisfies

$$
\lim _{\varepsilon \rightarrow 0} J_{\varepsilon}\left(\chi_{\Omega_{1 \varepsilon}} \Phi^{0}+\chi_{\Omega_{2 \varepsilon}} \Psi^{0}\right)=J_{0}\left(\Phi^{0}, \Psi^{0}\right)
$$

where $J_{0}$ is the functional given by (3.13).

Proof. Let $\psi_{\varepsilon}^{0}=\chi_{\Omega_{1 \varepsilon}} \Phi^{0}+\chi_{\Omega_{2 \varepsilon}} \Psi^{0}$ and $\psi_{\varepsilon}=\left(\psi_{1 \varepsilon}, \psi_{2 \varepsilon}\right)$ be the solution of the transposed problem (3.2) with final data $\chi_{\Omega_{1 \varepsilon}} \Phi^{0}+\chi_{\Omega_{2 \varepsilon}} \Psi^{0}$. Then, as in the proof of Proposition 6.6,

$$
\begin{aligned}
J_{\varepsilon}\left(\psi_{\varepsilon}^{0}\right)= & \frac{1}{2}\left(\int_{0}^{T} \int_{\omega_{1 \varepsilon}}\left|\psi_{1 \varepsilon}\right|^{2} \mathrm{~d} x \mathrm{~d} t+\int_{0}^{T} \int_{\omega_{2 \varepsilon}}\left|\psi_{2 \varepsilon}\right|^{2} \mathrm{~d} x \mathrm{~d} t\right)+\delta_{1}\left\|\Phi^{0}\right\|_{\mathrm{L}^{2}\left(\Omega_{1 \varepsilon}\right)} \\
& +\delta_{2}\left\|\Psi^{0}\right\|_{\mathrm{L}^{2}\left(\Omega_{2 \varepsilon}\right)}-\int_{\Omega_{1 \varepsilon}}\left(w_{\varepsilon}-v_{1 \varepsilon}(T)\right) \Phi^{0} \mathrm{~d} x-\int_{\Omega_{2 \varepsilon}}\left(w_{\varepsilon}-v_{2 \varepsilon}(T)\right) \Psi^{0} \mathrm{~d} x,
\end{aligned}
$$

where $v_{\varepsilon}=\left(v_{1 \varepsilon}, v_{2 \varepsilon}\right)$ is the solution of the auxiliary problem (3.3). Now, since

$$
\left(\chi_{\Omega_{1 \varepsilon}} \Phi^{0}, \chi_{\Omega_{2 \varepsilon}} \Psi^{0}\right) \rightarrow\left(\theta_{1} \Phi^{0}, \theta_{2} \Psi^{0}\right) \quad \text { weakly in }\left[\mathrm{L}^{2}(\Omega)\right]^{2},
$$

Theorem 2.2 applied to $\left(\psi_{1 \varepsilon}, \psi_{2 \varepsilon}\right)$ yields convergences (2.14), written for $z_{\varepsilon}=\psi_{\varepsilon}$ and $\left(z_{1}, z_{2}\right)$ equal to the solution $\left(\psi_{1}, \psi_{2}\right)$ of $(3.14)$. We need to pass to the limit in (6.20). For the first two terms, we write

$$
\begin{aligned}
I_{\varepsilon}= & \frac{1}{2}\left(\int_{0}^{T} \int_{\Omega_{1 \varepsilon}} \chi_{\omega}\left|\psi_{1 \varepsilon}\right|^{2} \mathrm{~d} x \mathrm{~d} t+\int_{0}^{T} \int_{\Omega_{2 \varepsilon}} \chi_{\omega}\left|\psi_{2 \varepsilon}\right|^{2} \mathrm{~d} x \mathrm{~d} t\right)=\frac{1}{2} \int_{0}^{T} \int_{\Omega_{1 \varepsilon}} \chi_{\omega}\left(\psi_{1 \varepsilon}-\psi_{1}\right) \psi_{1 \varepsilon} \mathrm{d} x \mathrm{~d} t \\
& +\frac{1}{2} \int_{0}^{T} \int_{\Omega_{1 \varepsilon}} \chi_{\omega} \psi_{1} \psi_{1 \varepsilon} \mathrm{d} x \mathrm{~d} t+\frac{1}{2} \int_{0}^{T} \int_{\Omega_{2 \varepsilon}} \chi_{\omega}\left(\psi_{2 \varepsilon}-\theta_{2}^{-1} \psi_{2}\right) \psi_{2 \varepsilon} \mathrm{d} x \mathrm{~d} t+\frac{1}{2} \int_{0}^{T} \int_{\Omega_{2 \varepsilon}} \chi_{\omega} \theta_{2}^{-1} \psi_{2} \psi_{2 \varepsilon} \mathrm{d} x \mathrm{~d} t .
\end{aligned}
$$


Observe that we can apply Theorem 2.5 to the problem solved by $\psi_{\varepsilon}$, since clearly $(2.25)$ holds true. Then, we can pass to the limit in the first and third terms of $I_{\varepsilon}$ using the Hölder's inequality to obtain

$$
\begin{aligned}
& \lim _{\varepsilon \rightarrow 0}\left(\frac{1}{2} \int_{0}^{T} \int_{\Omega_{1 \varepsilon}} \chi_{\omega}\left(\psi_{1 \varepsilon}-\psi_{1}\right) \psi_{1 \varepsilon} \mathrm{d} x \mathrm{~d} t+\frac{1}{2} \int_{0}^{T} \int_{\Omega_{2 \varepsilon}} \chi_{\omega}\left(\psi_{2 \varepsilon}-\theta_{2}^{-1} \psi_{2}\right) \psi_{2 \varepsilon} \mathrm{d} x \mathrm{~d} t\right) \\
& \leq \frac{1}{2} \lim _{\varepsilon \rightarrow 0}\left(\left\|\psi_{1 \varepsilon}-\psi_{1}\right\|_{\mathrm{C}^{0}\left(0, T ; \mathrm{L}^{2}\left(\Omega_{1 \varepsilon}\right)\right)}\left\|\psi_{1 \varepsilon}\right\|_{\mathrm{L}^{\infty}\left(0, T ; \mathrm{L}^{2}\left(\Omega_{1 \varepsilon}\right)\right)}\right) \\
& \quad+\frac{1}{2} \lim _{\varepsilon \rightarrow 0}\left(\left\|\psi_{2 \varepsilon}-\theta_{2}^{-1} \psi_{2}\right\|_{\mathrm{C}^{0}\left(0, T ; \mathrm{L}^{2}\left(\Omega_{2 \varepsilon}\right)\right)}\left\|\psi_{2 \varepsilon}\right\|_{\mathrm{L}^{\infty}\left(0, T ; \mathrm{L}^{2}\left(\Omega_{2 \varepsilon}\right)\right)}\right)=0 .
\end{aligned}
$$

Hence,

$$
\lim _{\varepsilon \rightarrow 0} I_{\varepsilon}=\lim _{\varepsilon \rightarrow 0}\left(\frac{1}{2} \int_{0}^{T} \int_{\omega} \psi_{1} \widetilde{\psi_{1 \varepsilon}} \mathrm{d} x \mathrm{~d} t+\frac{1}{2} \int_{0}^{T} \int_{\omega} \theta_{2}^{-1} \widetilde{\psi_{2}} \widetilde{\psi_{2 \varepsilon}} \mathrm{d} x \mathrm{~d} t\right)=\frac{1}{2} \int_{0}^{T} \int_{\omega}\left(\theta_{1}\left(\psi_{1}\right)^{2}+\theta_{2}^{-1}\left(\psi_{2}\right)^{2}\right) \mathrm{d} x \mathrm{~d} t .
$$

On the other hand, using (2.1) one obtains

$$
\lim _{\varepsilon \rightarrow 0} \delta_{1}\left(\int_{\Omega} \chi_{\Omega_{1 \varepsilon}}\left|\Phi^{0}\right|^{2} \mathrm{~d} x\right)^{\frac{1}{2}}+\lim _{\varepsilon \rightarrow 0} \delta_{2}\left(\int_{\Omega} \chi_{\Omega_{2 \varepsilon}}\left|\Psi^{0}\right|^{2} \mathrm{~d} x\right)^{\frac{1}{2}}=\delta_{1} \sqrt{\theta_{1}}\left\|\Phi^{0}\right\|_{\mathrm{L}^{2}(\Omega)}+\delta_{2} \sqrt{\theta_{2}}\left\|\Psi^{0}\right\|_{\mathrm{L}^{2}(\Omega)} .
$$

For the last two terms of (6.20), using (3.31) yields

$$
\begin{aligned}
& \lim _{\varepsilon \rightarrow 0}\left(\int_{\Omega_{1 \varepsilon}}\left(w_{\varepsilon}-v_{1 \varepsilon}(T)\right) \Phi^{0} \mathrm{~d} x+\int_{\Omega_{2 \varepsilon}}\left(w_{\varepsilon}-v_{2 \varepsilon}(T)\right) \Psi^{0} \mathrm{~d} x\right) \\
& =\lim _{\varepsilon \rightarrow 0}\left(\int_{\Omega_{1 \varepsilon}}\left[\left(w_{\varepsilon}-v_{1 \varepsilon}(T)\right)-\left(w-v_{1}(T)\right)\right] \Phi^{0} \mathrm{~d} x+\int_{\Omega}\left(w-v_{1}(T)\right) \chi_{\Omega_{1 \varepsilon}} \Phi^{0} \mathrm{~d} x\right) \\
& \quad+\lim _{\varepsilon \rightarrow 0}\left(\int_{\Omega_{2 \varepsilon}}\left[\left(w_{\varepsilon}-v_{2 \varepsilon}(T)\right)-\left(w-\theta_{2}^{-1} v_{2}(T)\right)\right] \Psi^{0} \mathrm{~d} x+\int_{\Omega}\left(w-\theta_{2}^{-1} v_{2}(T)\right) \chi_{\Omega_{2 \varepsilon}} \Psi^{0} \mathrm{~d} x\right) \\
& =\int_{\Omega}\left(w-v_{1}(T)\right) \theta_{1} \Phi^{0} \mathrm{~d} x+\int_{\Omega}\left(w-\theta_{2}^{-1} v_{2}(T)\right) \theta_{2} \Psi^{0} \mathrm{~d} x .
\end{aligned}
$$

Therefore, combining the above identities one gets the desired result.

The next proposition makes use of the following general statement proved in [7].

Lemma 6.8 [7]. Let $\mathcal{O}$ be an open set of $\mathbb{R}^{n}$ and $\left\{\mathcal{O}_{\varepsilon}\right\}_{\varepsilon} \subset \mathcal{O}$ a sequence of open subsets of $\mathcal{O}$. Suppose that $\left\{v_{\varepsilon}\right\}_{\varepsilon} \subset L^{p}\left(\mathcal{O}_{\varepsilon}\right), p>1$, is such that, as $\varepsilon \rightarrow 0$,

for some $v \in L^{p}(\mathcal{O})$. Then

$$
\left\{\begin{array}{l}
\chi_{\mathcal{O}_{\varepsilon}} \rightarrow \chi_{0} \text { weakly*in } \mathrm{L}^{\infty}(\mathcal{O}) \\
\widetilde{v_{\varepsilon}} \rightarrow \chi_{0} v \quad \text { weakly in } L^{p}(\mathcal{O})
\end{array}\right.
$$

$$
\liminf _{\varepsilon \rightarrow 0} \int_{\mathcal{O}_{\varepsilon}}\left|v_{\varepsilon}\right|^{p} \mathrm{~d} x \geq \int_{\mathcal{O}} \chi_{0}|v|^{p} \mathrm{~d} x
$$

Proposition 6.9. Under the assumptions of Propositions 6.6 and 6.7 (for the cases $-1<\gamma<1$ and $\gamma=1$, respectively), for any sequence $\left\{\psi_{\varepsilon}^{0}\right\}_{\varepsilon} \subset \mathrm{L}^{2}(\Omega)$ such that as $\varepsilon \rightarrow 0$,

$$
\left(\chi_{\Omega_{1 \varepsilon}} \psi_{\varepsilon}^{0}, \chi_{\Omega_{2 \varepsilon}} \psi_{\varepsilon}^{0}\right) \rightarrow\left(\theta_{1} \Phi^{0}, \theta_{2} \Psi^{0}\right) \quad \text { weakly in }\left[\mathrm{L}^{2}(\Omega)\right]^{2},
$$


for some $\left(\Phi^{0}, \Psi^{0}\right)$ in $\left[\mathrm{L}^{2}(\Omega)\right]^{2}$, we have

$$
\liminf _{\varepsilon \rightarrow 0} J_{\varepsilon}\left(\psi_{\varepsilon}^{0}\right) \geq J_{0}\left(\Phi^{0}, \Psi^{0}\right)
$$

where $J_{0}$ is defined by (3.8) and (3.13) for the cases $-1<\gamma<1$ and $\gamma=1$, respectively.

Proof. Let $\psi_{\varepsilon}$ be the solution of (3.2) with final data $\psi_{\varepsilon}^{0} \in \mathrm{L}^{2}(\Omega)$.

Case $-\mathbf{1}<\boldsymbol{\gamma}<\mathbf{1}$ : Let $J_{\varepsilon}$ be given by (3.4). From Theorem 2.2 and Proposition 2.4 applied to $\psi_{\varepsilon}$ we have

$$
\frac{1}{2} \lim _{\varepsilon \rightarrow 0} \int_{0}^{T} \int_{\omega}\left|\psi_{\varepsilon}\right|^{2} \mathrm{~d} x \mathrm{~d} t=\frac{1}{2} \int_{0}^{T} \int_{\omega}|\psi|^{2} \mathrm{~d} x \mathrm{~d} t
$$

where $\psi$ is the solution of the homogeneous transposed problem (3.9). Now, by (3.29) and (6.22), we get

$$
\lim _{\varepsilon \rightarrow 0} \int_{\Omega}\left(w_{\varepsilon}-v_{\varepsilon}(T)\right) \psi_{\varepsilon}^{0} \mathrm{~d} x=\int_{\Omega}(w-v(T))\left(\theta_{1} \Phi^{0}+\theta_{2} \Psi^{0}\right) \mathrm{d} x .
$$

Hence, using (2.1), (6.22), (6.24), (6.25) and Lemma 6.8, we get

$$
\begin{aligned}
\liminf _{\varepsilon \rightarrow 0} J_{\varepsilon}\left(\psi_{\varepsilon}^{0}\right) & \geq \frac{1}{2} \int_{0}^{T} \int_{\omega}|\psi|^{2} \mathrm{~d} x \mathrm{~d} t+\delta_{1} \sqrt{\theta_{1}}\left\|\Phi^{0}\right\|_{L^{2}(\Omega)}+\delta_{2} \sqrt{\theta_{2}}\left\|\Psi^{0}\right\|_{L^{2}(\Omega)} \\
& -\int_{\Omega}(w-v(T))\left(\theta_{1} \Phi^{0}+\theta_{2} \Psi^{0}\right) \mathrm{d} x=J_{0}\left(\Phi^{0}, \Psi^{0}\right) .
\end{aligned}
$$

Case $\gamma=1$ : By the definition of $J_{\varepsilon}$, we have

$$
\begin{aligned}
J_{\varepsilon}\left(\psi_{\varepsilon}^{0}\right)=\frac{1}{2}\left(\int_{0}^{T} \int_{\omega_{1 \varepsilon}}\left|\psi_{1 \varepsilon}\right|^{2} \mathrm{~d} x \mathrm{~d} t+\int_{0}^{T} \int_{\omega_{2 \varepsilon}}\left|\psi_{2 \varepsilon}\right|^{2} \mathrm{~d} x \mathrm{~d} t\right)+\delta_{1}\left\|\psi_{\varepsilon}^{0}\right\|_{\mathrm{L}^{2}\left(\Omega_{1 \varepsilon}\right)} \\
\quad+\delta_{2}\left\|\psi_{\varepsilon}^{0}\right\|_{\mathrm{L}^{2}\left(\Omega_{2 \varepsilon}\right)}-\int_{\Omega_{1 \varepsilon}}\left(w_{\varepsilon}-v_{1 \varepsilon}(T)\right) \psi_{\varepsilon}^{0} \mathrm{~d} x-\int_{\Omega_{2 \varepsilon}}\left(w_{\varepsilon}-v_{2 \varepsilon}(T)\right) \psi_{\varepsilon}^{0} \mathrm{~d} x
\end{aligned}
$$

For this case, Theorem 2.2 applied to problem of $\psi_{\varepsilon}$ gives

$$
\left\{\begin{array}{l}
(i) \widetilde{\psi_{1 \varepsilon}} \rightarrow \theta_{1} \psi_{1} \\
\text { (ii) } \overline{\psi_{2 \varepsilon}} \rightarrow \psi_{2}
\end{array}\right.
$$

where $\left(\psi_{1}, \psi_{2}\right)$ is the solution of (3.14). Now, from (2.1) and (6.27), applying Lemma 6.8 gives

$$
\liminf _{\varepsilon \rightarrow 0} \int_{0}^{T} \int_{\Omega_{1 \varepsilon}} \chi_{\omega}\left|\psi_{1 \varepsilon}\right|^{2} \mathrm{~d} x \mathrm{~d} t=\liminf _{\varepsilon \rightarrow 0} \int_{0}^{T} \int_{\Omega_{1 \varepsilon}}\left|\psi_{1 \varepsilon} \chi_{\omega}\right|^{2} \mathrm{~d} x \mathrm{~d} t \geq \int_{0}^{T} \int_{\omega} \theta_{1}\left|\psi_{1}\right|^{2} \mathrm{~d} x \mathrm{~d} t
$$

and

$$
\liminf _{\varepsilon \rightarrow 0} \int_{0}^{T} \int_{\Omega_{2 \varepsilon}} \chi_{\omega}\left|\psi_{2 \varepsilon}\right|^{2} \mathrm{~d} x \mathrm{~d} t \geq \int_{0}^{T} \int_{\omega} \theta_{2}\left|\frac{\psi_{2}}{\theta_{2}}\right|^{2} \mathrm{~d} x \mathrm{~d} t=\theta_{2}^{-1} \int_{0}^{T} \int_{\omega}\left|\psi_{2}\right|^{2} \mathrm{~d} x \mathrm{~d} t .
$$

On the other hand, by same argument used to prove (6.21),

$$
\begin{aligned}
& \lim _{\varepsilon \rightarrow 0}\left(\int_{\Omega_{1 \varepsilon}}\left(w_{\varepsilon}-v_{1 \varepsilon}(T)\right) \psi_{\varepsilon}^{0} \mathrm{~d} x+\int_{\Omega_{2 \varepsilon}}\left(w_{\varepsilon}-v_{2 \varepsilon}(T)\right) \psi_{\varepsilon}^{0} \mathrm{~d} x\right) \\
& =\lim _{\varepsilon \rightarrow 0}\left(\int_{\Omega_{1 \varepsilon}}\left[\left(w_{\varepsilon}-v_{1 \varepsilon}(T)\right)-\left(w-v_{1}(T)\right)\right] \psi_{\varepsilon}^{0} \mathrm{~d} x+\int_{\Omega}\left(w-v_{1}(T)\right) \chi_{\Omega_{1 \varepsilon}} \psi_{\varepsilon}^{0} \mathrm{~d} x\right) \\
& \quad+\lim _{\varepsilon \rightarrow 0}\left(\int_{\Omega_{2 \varepsilon}}\left[\left(w_{\varepsilon}-v_{2 \varepsilon}(T)\right)-\left(w-\theta_{2}^{-1} v_{2}(T)\right)\right] \psi_{\varepsilon}^{0} \mathrm{~d} x+\int_{\Omega}\left(w-\theta_{2}^{-1} v_{2}(T)\right) \chi_{\Omega_{2 \varepsilon}} \psi_{\varepsilon}^{0} \mathrm{~d} x\right) \\
& =\int_{\Omega}\left(w-v_{1}(T)\right) \theta_{1} \Phi^{0} \mathrm{~d} x+\int_{\Omega}\left(w-\theta_{2}^{-1} v_{2}(T)\right) \theta_{2} \Psi^{0} \mathrm{~d} x .
\end{aligned}
$$


Using Lemma 6.8 once again, we can pass to the limit for the remaining terms of (6.26). Hence, together with (6.28)-(6.30), one has the claimed result.

Thanks to the above propositions, we are now able to prove Theorems 6.4 and 3.5, our main result.

Proof of Theorem 6.4. By Remark 6.3, the sequence $\left\{\widehat{\varphi}_{\varepsilon}^{0}\right\}_{\varepsilon}$ of the minimum points of $J_{\varepsilon}$, satisfies (up to a subsequence), convergence (6.14) for some $\left(\xi^{0}, \nu^{0}\right) \in\left[\mathrm{L}^{2}(\Omega)\right]^{2}$. From Proposition 6.9, we have

$$
J_{0}\left(\xi^{0}, \nu^{0}\right) \leq \liminf _{\varepsilon \rightarrow 0} J_{\varepsilon}\left(\widehat{\varphi}_{\varepsilon}^{0}\right)
$$

Let us consider first the case $-1<\gamma<1$. Since $\widehat{\varphi}_{\varepsilon}^{0}$ is the minimum point of the functional $J_{\varepsilon}$, for any $\left(\Phi^{0}, \Psi^{0}\right) \in\left[\mathrm{L}^{2}(\Omega)\right]^{2}$, using Proposition 6.6 we have,

$$
\limsup _{\varepsilon \rightarrow 0} J_{\varepsilon}\left(\widehat{\varphi}_{\varepsilon}^{0}\right) \leq \limsup J_{\varepsilon}\left(\chi_{\Omega_{1 \varepsilon}} \Phi^{0}+\chi_{\Omega_{2 \varepsilon}} \Psi^{0}\right)=J_{0}\left(\Phi^{0}, \Psi^{0}\right)
$$

From $(6.31),(6.32)$ and Theorem 3.3 , we get $\left(\xi^{0}, \nu^{0}\right)=\left(\widehat{\Phi}^{0}, \widehat{\Psi}^{0}\right)$ where $\left(\widehat{\Phi}^{0}, \widehat{\Psi}^{0}\right)$ is the unique minimum point of the functional $J_{0}$ given in (3.8) and consequently, the whole sequence in (6.14) converges.

Now, for the case $\gamma=1$, using Proposition 6.7 and the fact that $\widehat{\varphi}_{\varepsilon}^{0}$ is the minimum point of $J_{\varepsilon}$, we obtain again (6.32). This, together with (6.31) and Theorem 3.4, shows that $\left(\xi^{0}, \nu^{0}\right)=\left(\widehat{\Phi}^{0}, \widehat{\Psi}^{0}\right)$, the unique minimum point of the functional $J_{0}$ given in (3.13) and again the whole sequence in (6.14) converges.

Proof of Theorem 3.5. Observe that the convergences in (3.18) and (3.25) are direct consequences of Theorem 6.4 and Corollary 6.5. On the other hand, (3.17)(i) and (3.18)(i) (see also Rem. 2.6) together with the corrector result given in Theorem 2.5 for the case $-1<\gamma<1$ imply (3.19)(ii). Also, Theorem 2.2 applies to problem (3.5) which proves (3.19)(i) and (iii). Meanwhile for the case $\gamma=1$, using assumption (3.24)(i) and convergences (3.25)(i) and (ii) with Theorem 2.2, we have (3.26). Consequently, the controllability results for the homogenized problems proved in Theorems 3.3 and 3.4 yield (3.21) and (3.28), respectively.

It remains to prove, for the case $-1<\gamma<1$, convergences (3.22) and (3.23).

Observe that using (6.31) and (6.32), we deduce that

$$
J_{0}\left(\xi^{0}, \nu^{0}\right)=J_{0}\left(\widehat{\Phi}^{0}, \widehat{\Psi}^{0}\right) \leq \liminf _{\varepsilon \rightarrow 0} J_{\varepsilon}\left(\widehat{\varphi}_{\varepsilon}^{0}\right) \leq \limsup _{\varepsilon \rightarrow 0} J_{\varepsilon}\left(\widehat{\varphi}_{\varepsilon}^{0}\right) \leq J_{0}\left(\widehat{\Phi}^{0}, \widehat{\Psi}^{0}\right) .
$$

This gives

$$
\lim _{\varepsilon \rightarrow 0} J_{\varepsilon}\left(\widehat{\varphi}_{\varepsilon}^{0}\right)=J_{0}\left(\widehat{\Phi}^{0}, \widehat{\Psi}^{0}\right) .
$$

Now, if $\widehat{\varphi}_{\varepsilon}=\left(\widehat{\varphi}_{1 \varepsilon}, \widehat{\varphi}_{2 \varepsilon}\right)$ is the control given in Theorem 3.2 and $\widehat{\varphi}$ is the solution of (3.9), then writing (6.24) for $\widehat{\varphi}_{\varepsilon}$ and using Corollary 6.5 one obtains

$\lim _{\varepsilon \rightarrow 0} \frac{1}{2}\left(\int_{0}^{T} \int_{\omega_{1 \varepsilon}}\left|\widehat{\varphi}_{1 \varepsilon}\right|^{2} \mathrm{~d} x \mathrm{~d} t+\int_{0}^{T} \int_{\omega_{1 \varepsilon}}\left|\widehat{\varphi}_{2 \varepsilon}\right|^{2} \mathrm{~d} x \mathrm{~d} t\right)=\frac{1}{2} \lim _{\varepsilon \rightarrow 0} \int_{0}^{T} \int_{\omega} \widetilde{\mid \widehat{\varphi}_{1 \varepsilon}}+\left.\widetilde{\widehat{\varphi}_{2 \varepsilon}}\right|^{2} \mathrm{~d} x \mathrm{~d} t=\frac{1}{2} \int_{0}^{T} \int_{\omega}|\widehat{\varphi}|^{2} \mathrm{~d} x \mathrm{~d} t$.

Furthermore, using (3.29) and (3.18)(ii), we get

$$
\lim _{\varepsilon \rightarrow 0}\left(\int_{\Omega_{1 \varepsilon}}\left(w_{\varepsilon}-v_{1 \varepsilon}(T)\right) \widehat{\varphi}_{\varepsilon}^{0} \mathrm{~d} x+\int_{\Omega_{2 \varepsilon}}\left(w_{\varepsilon}-v_{2 \varepsilon}(T)\right) \widehat{\varphi}_{\varepsilon}^{0} \mathrm{~d} x\right) \int_{\Omega}(w-v(T))\left(\theta_{1} \widehat{\Phi}^{0}+\theta_{2} \widehat{\Psi}^{0}\right) \mathrm{d} x .
$$

Taking into account these convergences, we have (3.22). Clearly, if $\delta_{1}=\delta_{2}$, we get (3.23). 


\section{Appendix by Ammar Kohdja}

We state here the unique continuation result for the coupled problem (3.14) (for which the results from [12] do not apply). We employed it in this paper twice, for the case $\gamma=1$. First, we used it in the proof of the controllability of the homogenized problem (Thm. 3.4) and second, in that of the uniform estimate (6.1).

When inquiring about a possible unique continuation principle for that problem, we addressed the question to Farid Ammar Kohdja, who has given a positive answer and suggested us the proof below. The authors are grateful and indebted to him for the proof and for the time he spent in proving the result.

Theorem 7.1 [1]. Under the assumptions of Theorem 3.4, let $\varphi=\left(\varphi_{1}, \varphi_{2}\right)$ be the solution of (3.14). If $\varphi$ is zero in $\omega \times(0, T)$ then $\varphi$ is zero in $\Omega \times(0, T)$.

Proof. We define the operator $L$ and the adjoint one by

$$
L=\left(\begin{array}{cc}
\frac{1}{\theta_{1}} \Delta-c_{h} \frac{\theta_{2}}{\theta_{1}} & \frac{c_{h}}{\theta_{1}} \\
c_{h} \theta_{2} & -c_{h}
\end{array}\right), \quad L^{*}=\left(\begin{array}{cc}
\frac{1}{\theta_{1}} \Delta-c_{h} \frac{\theta_{2}}{\theta_{1}} c_{h} \theta_{2} \\
\frac{c_{h}}{\theta_{1}} & -c_{h}
\end{array}\right), \quad D(L)=D\left(L^{*}\right)=\left(H^{2}(\Omega) \cap H_{0}^{1}(\Omega)\right) \times L^{2}(\Omega)
$$

where $\Delta: L^{2}(\Omega) \rightarrow L^{2}(\Omega)$ is the usual unbounded Laplace operator with domain $H^{2}(\Omega) \cap H_{0}^{1}(\Omega)$. For simplicity, we prove the results for the case $A^{0}=I d$ and we can use the fact that all the eigenvalues of $-\Delta$ are simple. If not, it is sufficient to work on the eigenspaces, which have all finite dimension.

Let $0<\mu_{1}<\mu_{2}<\cdots<\mu_{k} \uparrow \infty$ be the sequence of the eigenvalues of $-\Delta$ and $\left(\varphi_{k}\right)$ the sequence of the corresponding normalized eigenvectors, which forms an orthonormal basis in $L^{2}(\Omega)$.

Then, since $\theta_{1}, \theta_{2}$ are positive constants and $\theta_{1}+\theta_{2}=1$, the eigenvalues of $L$ verify the following equation:

$$
p(\lambda)=\theta_{1} \lambda^{2}+\left(\mu_{k}+c_{h}\right) \lambda+c_{h} \mu_{k}=0, k \geq 1 .
$$

Since $\left(\mu_{k}+c_{h}\right)^{2}-4 \theta_{1} c_{h} \mu_{k}>0$ for all $k \geq 1$, these eigenvalues are real negative numbers given by

$$
\lambda_{k}^{ \pm}=\frac{-\mu_{k}-c_{h} \pm \sqrt{\left(\mu_{k}+c_{h}\right)^{2}-4 \theta_{1} c_{h} \mu_{k}}}{2 \theta_{1}} .
$$

Moreover: $p\left(-c_{h}\right)=\theta_{1} c_{h}^{2}-\left(\mu_{k}+c_{h}\right) c_{h}+c_{h} \mu_{k}=-\theta_{2} c_{h}^{2}<0$, so that $\lambda_{k}^{-}<-c_{h}<\lambda_{k}^{+}$.

It is readily seen that the sequences $\left(\lambda_{k}^{+}\right)$and $\left(\lambda_{k}^{-}\right)$are decreasing and as $k \rightarrow \infty$ :

$$
\lambda_{k}^{-} \searrow-\infty \quad \text { and } \quad \lambda_{k}^{+} \sim-c_{h} \frac{\mu_{k}}{\left(\mu_{k}+c_{h}\right)} \underset{k \rightarrow \infty}{\searrow}-c_{h} .
$$

Then, we can order the eigenvalues as follows:

$$
\lambda_{1}^{+}>\lambda_{2}^{+}>\cdots>-c_{h}>\lambda_{1}^{-}>\lambda_{2}^{-}>\cdots>\lambda_{k}^{-} \searrow-\infty .
$$

The associated family of eigenvectors $\left(\Phi_{k}^{ \pm}\right)$is given by $\Phi_{k}^{ \pm}=\rho_{k}^{ \pm}\left(\begin{array}{c}\lambda_{k}^{ \pm}+c_{h} \\ c_{h} \theta_{2}\end{array}\right) \varphi_{k}, k \geq 1$, and if we set $\Psi_{k}^{ \pm}=\rho_{k}^{ \pm}\left(\begin{array}{c}\lambda_{k}^{ \pm}+c_{h} \\ \frac{c_{h}}{\theta_{1}}\end{array}\right) \varphi_{k}$, with $\rho_{k}^{ \pm}$a normalizing sequence, then $\left(\Phi_{k}^{ \pm}\right)$and $\left(\Psi_{k}^{ \pm}\right)$form biorthogonal families, i.e.

$$
\left\langle\Phi_{k}^{ \pm}, \Psi_{j}^{ \pm}\right\rangle_{L^{2}}=\delta_{k j},\left\langle\Phi_{k}^{ \pm}, \Psi_{j}^{\mp}\right\rangle_{L^{2}}=0, k, j \geq 1 .
$$

To do that we choose $\left(\rho_{k}^{ \pm}\right)^{2}=\frac{1}{\left(\lambda_{k}^{ \pm}+c_{h}\right)^{2}+c_{h}^{2} \frac{\theta_{2}}{\theta_{1}}}$. 
Let us now prove that $\left(\Phi_{k}^{ \pm}\right)$is a Riesz basis. To do that, we have to prove the inequality

$$
\mu_{1} \sum_{k=1}^{N}\left(\left(a_{k}^{+}\right)^{2}+\left(a_{k}^{-}\right)^{2}\right) \leq\left|\sum_{k=1}^{N} a_{k}^{ \pm} \Phi_{k}^{ \pm}\right|_{L^{2}}^{2} \leq \mu_{2} \sum_{k=1}^{N}\left(\left(a_{k}^{+}\right)^{2}+\left(a_{k}^{-}\right)^{2}\right),
$$

for any $N \geq 1$ and for every sequence $\left(a_{k}\right)$, where $\mu_{i}, i=1,2$ are two strictly positive constants independent of $N$ and $\left(a_{k}\right)$.

Using the fact that the spaces $\operatorname{Span}\left\langle\Phi_{k}^{+}, \Phi_{k}^{-}\right\rangle$are pairwise orthogonal, it suffices to prove the inequality for $\left|a_{k}^{+} \Phi_{k}^{+}+a_{k}^{-} \Phi_{k}^{-}\right|_{L^{2}}^{2}$, which is given by

$$
\left|a_{k}^{+} \Phi_{k}^{+}+a_{k}^{-} \Phi_{k}^{-}\right|_{L^{2}}^{2}=\left(a_{k}^{+}\right)^{2}\left|\Phi_{k}^{+}\right|_{L^{2}}^{2}+2\left\langle\Phi_{k}^{+}, \Phi_{k}^{-}\right\rangle_{L^{2}} a_{k}^{+} a_{k}^{-}+\left|\Phi_{k}^{-}\right|_{L^{2}}^{2}\left(a_{k}^{-}\right)^{2} .
$$

Observe that

$$
\left|\Phi_{k}^{ \pm}\right|_{L^{2}}^{2}=\left(\rho_{k}^{ \pm}\right)^{2}\left(\left(\lambda_{k}^{ \pm}+c_{h}\right)^{2}+c_{h}^{2} \theta_{2}^{2}\right)=\frac{\left(\lambda_{k}^{ \pm}+c_{h}\right)^{2}+c_{h}^{2} \theta_{2}^{2}}{\left(\lambda_{k}^{ \pm}+c_{h}\right)^{2}+c_{h}^{2} \frac{\theta_{2}}{\theta_{1}}}
$$

Using (7.2) this gives

$$
\lim _{k \rightarrow \infty}\left|\Phi_{k}^{+}\right|_{L^{2}}^{2}=\theta_{1} \theta_{2}, \lim _{k \rightarrow \infty}\left|\Phi_{k}^{-}\right|_{L^{2}}^{2}=1
$$

On the other hand,

$$
\begin{aligned}
\left\langle\Phi_{k}^{+}, \Phi_{k}^{-}\right\rangle_{L^{2}} & =\rho_{k}^{+} \rho_{k}^{-}\left(\left(\lambda_{k}^{+}+c_{h}\right)\left(\lambda_{k}^{-}+c_{h}\right)+c_{h}^{2} \theta_{2}^{2}\right)=\rho_{k}^{+} \rho_{k}^{-}\left(\lambda_{k}^{+} \lambda_{k}^{-}+\left(\lambda_{k}^{+}+\lambda_{k}^{-}\right) c_{h}+\left(1+\theta_{2}^{2}\right) c_{h}^{2}\right) \\
& =\rho_{k}^{+} \rho_{k}^{-}\left(\frac{c_{h} \mu_{k}}{\theta_{1}}-\frac{\mu_{k}+c_{h}}{\theta_{1}} c_{h}+\left(1+\theta_{2}^{2}\right) c_{h}^{2}\right) \\
& =\frac{\left(1+\theta_{2}^{2}-\frac{1}{\theta_{1}}\right) c_{h}^{2}}{\sqrt{\left(\lambda_{k}^{+}+c_{h}\right)^{2}+c_{h}^{2} \frac{\theta_{2}}{\theta_{1}}} \sqrt{\left(\lambda_{k}^{-}+c_{h}\right)^{2}+c_{h}^{2} \frac{\theta_{2}}{\theta_{1}}}} \sim \frac{\left(1+\theta_{2}^{2}-\frac{1}{\theta_{1}}\right) c_{h}}{\sqrt{\frac{\theta_{2}}{\theta_{1}}}\left|\lambda_{k}^{-}\right|},
\end{aligned}
$$

where we used again (7.2). Hence, setting $\left.\tau=\frac{\left(1+\theta_{2}^{2}-\frac{1}{\theta_{1}}\right) c_{h}}{\sqrt{\frac{\theta_{2}}{\theta_{1}}}}\right)$ we have, asymptotically,

$$
\left|a_{k}^{+} \Phi_{k}^{+}+a_{k}^{-} \Phi_{k}^{-}\right|_{L^{2}}^{2}=\theta_{1} \theta_{2}\left(a_{k}^{+}\right)^{2}-2 \frac{\tau}{\lambda_{k}^{-}} a_{k}^{+} a_{k}^{-}+\left(a_{k}^{-}\right)^{2}
$$

Since in view of (7.2), one can find a $\delta>0$ such that, for $k$ large enough, $\left(\frac{\tau}{\lambda_{k}^{-}}\right)^{2}-\theta_{1} \theta_{2} \leq-\delta<0$ there exist $\mu_{1}, \mu_{2}>0$ (independent of $k$ ) such that

$$
\mu_{1}\left(\left(a_{k}^{+}\right)^{2}+\left(a_{k}^{-}\right)^{2}\right) \leq\left|a_{k}^{+} \Phi_{k}^{+}+a_{k}^{-} \Phi_{k}^{-}\right|_{L^{2}}^{2} \leq \mu_{2}\left(\left(a_{k}^{+}\right)^{2}+\left(a_{k}^{-}\right)^{2}\right),
$$

which implies that $\left\{\Phi_{k}^{+}, \Phi_{k}^{-}\right\}$is a Riesz basis. Then, the solution of the adjoint problem is

$$
\varphi=\sum_{k \geq 1} e^{\lambda_{k}^{ \pm} t}\left\langle\varphi^{0}, \Psi_{k}^{ \pm}\right\rangle \Phi_{k}^{ \pm}=\sum_{k \geq 1} e^{\lambda_{k}^{+} t}\left\langle\varphi^{0}, \Psi_{k}^{+}\right\rangle \Phi_{k}^{+}+\sum_{k \geq 1} e^{\lambda_{k}^{-} t}\left\langle\varphi^{0}, \Psi_{k}^{-}\right\rangle \Phi_{k}^{-}
$$

and the condition $\varphi=0$ in $(0, T) \times \omega$ takes the form

$$
\sum_{k \geq 1} e^{\lambda_{k}^{+} t}\left\langle\varphi^{0}, \Psi_{k}^{+}\right\rangle \Phi_{k}^{+}+\sum_{k \geq 1} e^{\lambda_{k}^{-} t}\left\langle\varphi^{0}, \Psi_{k}^{-}\right\rangle \Phi_{k}^{-}=0,(0, T) \times \omega .
$$


Extending (by analyticity) in time to $(0, \infty)$, we obtain

$$
\sum_{k \geq 1} e^{\lambda_{k}^{+} t}\left\langle\varphi^{0}, \Psi_{k}^{+}\right\rangle \Phi_{k}^{+}+\sum_{k \geq 1} e^{\lambda_{k}^{-} t}\left\langle\varphi^{0}, \Psi_{k}^{-}\right\rangle \Phi_{k}^{-}=0,(0, \infty) \times \omega .
$$

Multiplying this identity by $e^{-\lambda_{1} t}$ and letting $t \rightarrow \infty$, we get $\left\langle\varphi^{0}, \Psi_{1}^{+}\right\rangle \Phi_{1}^{+}=0$, in $\omega$. Hence, by induction, $\left\langle\varphi^{0}, \Psi_{k}^{ \pm}\right\rangle \Phi_{k}^{ \pm}=0$, in $\omega, \forall k \geq 1$. Since the eigenfunctions $\varphi_{k}$ are analytic, $\left\langle\varphi^{0}, \Psi_{k}^{ \pm}\right\rangle=0, \forall k \geq 1$, which implies $\varphi=0$ in $(0, T) \times \Omega$ and ends the proof.

Acknowledgements. The authors are grateful to Emmanuel Trélat for several enriching discussions about the unique continuation property. They also thank Claude Bardos and Jérôme Le Rousseau for helpful related suggestions.

\section{REFERENCES}

[1] F. Ammar Khodja, Personal communication.

[2] H.S. Carslaw and J.C. Jaeger, Conduction of Heat in Solids. The Clarendon Press, Oxford (1947).

[3] D. Cioranescu and P. Donato, An Introduction to Homogenization. Vol. 17 of Oxf. Lect. Ser. Math. Appl. Oxford Univ. Press, New York (1999).

[4] D. Cioranescu and J. Saint Jean Paulin, Homogenization in Open Sets with Holes. J. Math. Anal. Appl. 71 (1979) 590-607.

[5] D. Cioranescu, P. Donato and E. Zuazua, Approximate Controllability for the Wave Equation with Oscillating Coefficients. Proc. of IFLP Conf. 1990. Lect. Notes Control Inf. Sci. Springer-Verlag (1992) 118-124.

[6] P. Donato and E. Jose, Corrector Results for a Parabolic Problem with a Memory Effect. ESAIM: M2AN 44 (2010) $421-454$.

[7] P. Donato and A. Nabil, Approximate Controllability of Linear Parabolic Equations in Perforated Domains. ESAIM: COCV 6 (2001) 21-38.

[8] L. Faella and S. Monsurrò, Memory Effects Arising in the Homogenization of Composites with Inclusions. Topics on Mathematics for Smart Systems. World Sci. Publ., Hackensack, NJ (2007) 107-121.

[9] C. Fabre, J.P. Puel and E. Zuazua, Approximate Controllability for the Semilinear Heat Equation. Proc. Roy. Soc. Edinb. Sect. A 125 (1995) 31-61.

[10] E. Jose, Homogenization of a Parabolic Problem with an Imperfect Interface. Rev. Roum. Math. Pures Appl. 54 (2009) $189-222$.

[11] J.-L. Lions, Remarques sur la controlabilité approchée. In Proc. of Jornadas Hispano-Francesas sobre Control de Sistemas Distribuidos, octubre 1990. Grupo de Análisis Matemático Aplicado de la University of Malaga, Spain (1991) 77-87.

[12] J.-C. Saut and B. Scheurer, Unique Continuation for Some Evolution Equations. J. Diff. Eq. 66 (1987) $118-139$.

[13] E. Zuazua, Approximate Controllability for Linear Parabolic Equations with Rapidly Oscillating Coefficients. Control Cybernet. 4 (1994) 793-801.

[14] E. Zuazua, Controllability of Partial Differential Equations and its Semi-Discrete Approximations. Discr. Contin. Dynam. Syst. 8 (2002) 469-513. 